\title{
高镍三元正极材料的包覆与掺杂改性研究进展
}

\author{
柏祥涛 ${ }^{1}$, 班丽卿 ${ }^{2}$, 庄卫东 ${ }^{2}$
}

(1. 国联汽车动力电池研究院有限责任公司, 北京 101407 ; 2. 北京有色金属研究总院, 北京 100088)

摘 要: 随着新能源汽车的加速发展, 镍钴锰/铝酸锂三元正极材料、特别是高镍(镍含量大于 $50 \%$ )材料作为后起之 秀, 由于其性能和成本的综合指标优于传统的钴酸锂和磷酸铁锂, 引起了学术界和产业界极大的研究兴趣。但是受 其本身晶体结构和表面结构的限制, 三元正极材料也存在安全性较差、循环稳定性不足等缺点。近年来, 科研工作 者为解决这些问题、并进一步提升三元材料的性能, 在材料改性技术方面开展了大量工作，取得了令人瞩目的研究 成果。本文从改性元素对三元正极材料结构以及对电化学性能改善的机理出发, 介绍了包覆和掺杂两种改性技术的 研究进展, 并在此基础上对三元正极材料的发展方向做出展望。

关 键 词: 镍钴锰酸锂; 包覆; 掺杂; 机理

中图分类号: 0646 文献标识码: A

\section{Research Progress on Coating and Doping Modification of Nickel Rich Ternary Cathode Materials}

\author{
BAI Xiangtao $^{1}$, BAN Liqing ${ }^{2}$, ZHUANG Weidong ${ }^{2}$ \\ (1. China Automotive Battery Research Institute Co., Ltd, Beijing 101407, China; 2. General Research Institute for Nonferrous \\ Metals, Beijing 100088, China)
}

\begin{abstract}
In recent years, the development of new energy vehicles industry is accelerating. Lithium nickel cobalt manganese/aluminum oxide ternary cathode materials (NCM/NCA), especially with the nickel content $\geqslant 50 \%$, has aroused great interest in both academia and industry. This is mainly due to the fact that the aggregative parameters of performance and cost of $\mathrm{NCM} / \mathrm{NCA}$ are superior to those of traditional cathode materials, such as $\mathrm{LiCoO}_{2}$ and $\mathrm{LiFePO}_{4}$. However, the application of NCM/NCA is affected by a number of drawbacks, including poor safety and insufficient cycle stability and so on, which are mainly attributed to its crystal and surface structure. Researchers have carried out various efforts to solve these problems and further improve the performance of NCM/NCA. Some remarkable results have been achieved in the past few years. In this review, the latest research progress on coating and doping of Ni-rich ternary cathode materials is summarized from the view on the mechanism of structural and electrochemical improvement of NCM/NCA. Finally, the perspective for the development of NCM/NCA cathode materials is also prospected.
\end{abstract}

Key words: lithium nickel cobalt manganese oxide; coating; doping; mechanism

新能源汽车产业是国家战略新兴产业, 随着车 前途的储能介质受到了广泛关注 ${ }^{[1]}$ 与消费类电子 用动力电池需求的不断升级, 锂离子电池作为最有 用锂电池不同，车用动力电池有更为严苛的要求，

收稿日期: 2019-11-07; 收到修改稿日期：2020-01-14

基金项目: 国家重点研发计划一新能源专项(2016YFB0100400)

The National Key R\&D Program of China-New Energy Special Project (2016YFB0100400)

作者简介: 柏祥涛(1983-), 男, 博士研究生, 高级工程师. E-mail: baixt@glabat.com BAI Xiangtao (1983-), male, PhD candidate, senior engineer. E-mail: baixt@glabat.com 
如成本、能量密度、安全性、使用寿命等。研究 ${ }^{[2]}$ 表明，正极材料对这些性能有决定性作用。在众多 正极材料中, 镍钴锰/ 铝酸锂三元正极材料 $\left(\mathrm{LiNi}_{x} \mathrm{Co}_{y} \mathrm{Mn}_{1-x-y} \mathrm{O}_{2} / \mathrm{LiNi}_{x} \mathrm{Co}_{y} \mathrm{Al}_{1-x-y} \mathrm{O}_{2}\right.$, 简称 $\left.\mathrm{NCM} / \mathrm{NCA}\right) 、$ 特别是高镍 $\mathrm{NCM} / \mathrm{NCA}(0.5 \leqslant x<1)$ 被寄予厚望 ${ }^{[3-4]}$ 。 在此类材料中, 其放电比容量随着镍含量的增加而 升高 ${ }^{[1]}$, 但镍含量的增加也带来一系列新问题, 主 要可分为表面问题和体相问题 ${ }^{[2-7]}$, 如表面残碱、过 渡金属溶解、阳离子混排等。

在三元材料制备和存储过程中, 由于 $\mathrm{Ni}^{3+}$ 的不 稳定性, 其表面容易形成诸如 $\mathrm{LiOH}$ 和 $\mathrm{Li}_{2} \mathrm{CO}_{3}$ 之类 的碱性物质(可称为“残碱”), 残碱对三元材料的使 用和性能均有不利影响 ${ }^{[8]}$ 。 $\mathrm{LiOH}$ 使 $\mathrm{pH}$ 升高, 导致 匀浆过程中容易形成“果冻”而无法涂布。 $\mathrm{Li}_{2} \mathrm{CO}_{3}$ 造 成水含量超标, 同时还导致电池循环过程产气, 影 响安全性能。当 $\mathrm{Ni}$ 含量 $\leqslant 60 \%$ 时，通过一次烧结基 本可以将残碱控制在可接受的范围内, 但是当 $\mathrm{Ni}$ 含 量 $\geqslant 80 \%$ 时，则需对材料进行额外处理和多次烧结 [9-11]。除残碱外，活性材料与电解液之间的副反应也 是影响 NCM 性能的重要因素。依据密度泛函理论 (DFT) 计算表明 ${ }^{[12]}$, 当电解液与正极材料接触时, 电解液中的碳酸乙烯酯(EC)与 $\mathrm{NCM}$ 的表面立即发 生反应, 即此反应是化学反应而非电化学反应。在 电解液中普遍存在的微量 HF 会侵蚀正极材料导致 过渡金属溶解 ${ }^{[13]}$, 进而影响 NCM 的循环性能。

由于 $\mathrm{Li}^{+}(0.076 \mathrm{~nm})$ 与 $\mathrm{Ni}^{2+}(0.069 \mathrm{~nm})$ 的离子半 径十分接近, 因此晶格中的 $\mathrm{Li} 、 \mathrm{Ni}$ 很容易互换位置, 生成非化学计量比的结构, 即发生 $\mathrm{Li} / \mathrm{Ni}$ 混排 ${ }^{[14]}$ 。 $\mathrm{Ni}^{2+}$ 进入锂离子层后能够阻断 $\mathrm{Li}^{+}$的传输路径, 减少 可以参加充放电循环的 $\mathrm{Li}^{+}$的数量, 导致材料比容 量降低。当 $\mathrm{Li}^{+}$混排进入过渡金属层时，由于其半径 比过渡金属离子大，过渡金属层会因空间效应和正 电荷之间的互相排斥而膨胀，导致层间距扩大并挤 压相邻的锂离子层, 被压缩的锂离子层间距会降低 锂离子的扩散系数, 影响材料的倍率性能。此外, $\mathrm{Li} / \mathrm{Ni}$ 混排还对正极材料性能有其他不利的影响, 如结构不稳定 ${ }^{[14]}$ 、表面副反应增多 ${ }^{[15-16]}$ 等。

三元材料主要由 $\mathrm{Ni}^{2+} / \mathrm{Ni}^{3+}$ 和 $\mathrm{Ni}^{3+} / \mathrm{Ni}^{4+}$ 氧化还原 电对提供容量, 因此在充放电过程中, $\mathrm{Ni}$ 通常处于 不稳定的高价态 $\left(\mathrm{Ni}^{3+} 、 \mathrm{Ni}^{4+}\right)$, 容易通过失氧的方式 向稳定的低价态转变, 对应层状相-尖晶石相一岩盐 相的相转变过程 ${ }^{[5,17]}$ 。层状和岩盐相中仅有一个八 面体结构，而尖晶石相中则同时有四面体和八面体 结构。在由层状向尖晶石相转变的过程中, 需要部 分金属离子由八面体结构迁移至四面体结构中，处 于不稳定状态的高价 $\mathrm{Ni}$ 离子优先发生迁移。但由于
$\mathrm{Ni}^{3+}$ 和 $\mathrm{Ni}^{4+}$ 分别有 7 个和 6 个 $\mathrm{d}$ 轨道电子，根据晶体 场理论, 其处于八面体位置比四面体位置更稳定, 因此当温度升高时, $\mathrm{Ni}$ 离子更倾向于迁移至八面体 位置，即发生尖晶石向岩盐相的相转变，同时释放 氧气 ${ }^{[18]}$ 。随着镍含量的增加, 第一次相转变温度逐 渐降低，当镍含量由 $40 \%$ (NCM433, 指 $\mathrm{Ni} 、 \mathrm{Co}$ 、 $\mathrm{Mn}$ 的摩尔比为 $4: 3: 3$, 如无特殊说明, 下同)增加 到 $80 \%$ (NCM811) 时，温度由 $250{ }^{\circ} \mathrm{C}$ 降低到 $\sim 150{ }^{\circ} \mathrm{C}$ (图 1) ${ }^{[19]}$ 。有研究表明, 充电过程中 NCM523 的相变主要发生在材料的表面，生成岛状分布的尖 晶石结构, 但是当截止电压较高时 $(4.8 \mathrm{~V})$, 也会出 现岩盐相(图 2) ${ }^{[20]}$ 。

为解决以上问题，包覆和掺杂是两种有效的手 段。包覆改性一方面可以在材料表面沉积一层惰性 物质, 隔离正极材料与电解液之间的接触, 减少副 反应的发生; 另一方面也可以沉积一层活性材料, 减 少残碱的同时还可以降低表面电阻。掺杂改性一方 面可以通过改变材料的晶格常数或部分元素的价态 来提高材料结构的稳定性, 另一方面也可以通过降 低阳离子混排来提高材料的电子电导率和离子电导 率。迄今为止，已有不少文章 ${ }^{[21-23]}$ 对三元材料的制 备和改性进行了综述，尽管这些综述很好地介绍了 改性元素的种类和对电化学性能提升的效果, 但针 对包覆和掺杂元素对其结构改善的原理以及对电化
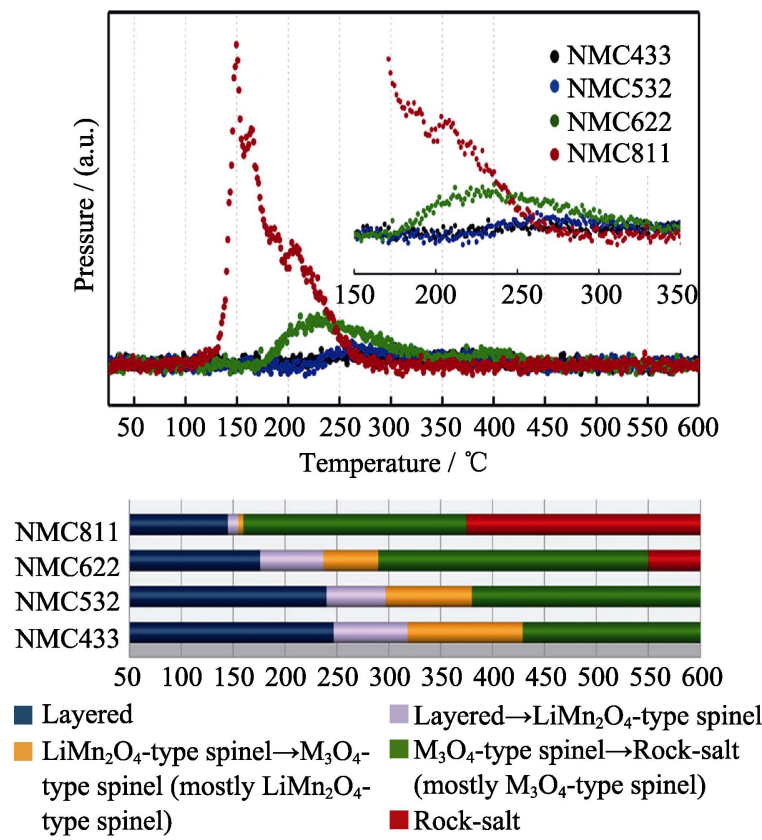

图 1 时间分辨 $\mathrm{XRD}(\mathrm{TR}-\mathrm{XRD})$ 测试过程中氧的质谱分析 $\left(\mathrm{O}_{2}, m / z=32\right)$ (上)及 $\mathrm{NCM}$ 相变温度区域(下 $)^{[19]}$

Fig. 1 Mass spectroscopy profiles for the oxygen $\left(\mathrm{O}_{2}, \mathrm{~m} / z=32\right)$ collected simultaneously during measurement of TR-XRD (upper panel) and the corresponding temperature region of the phase transitions for NCM (lower panel) ${ }^{[19]}$ 


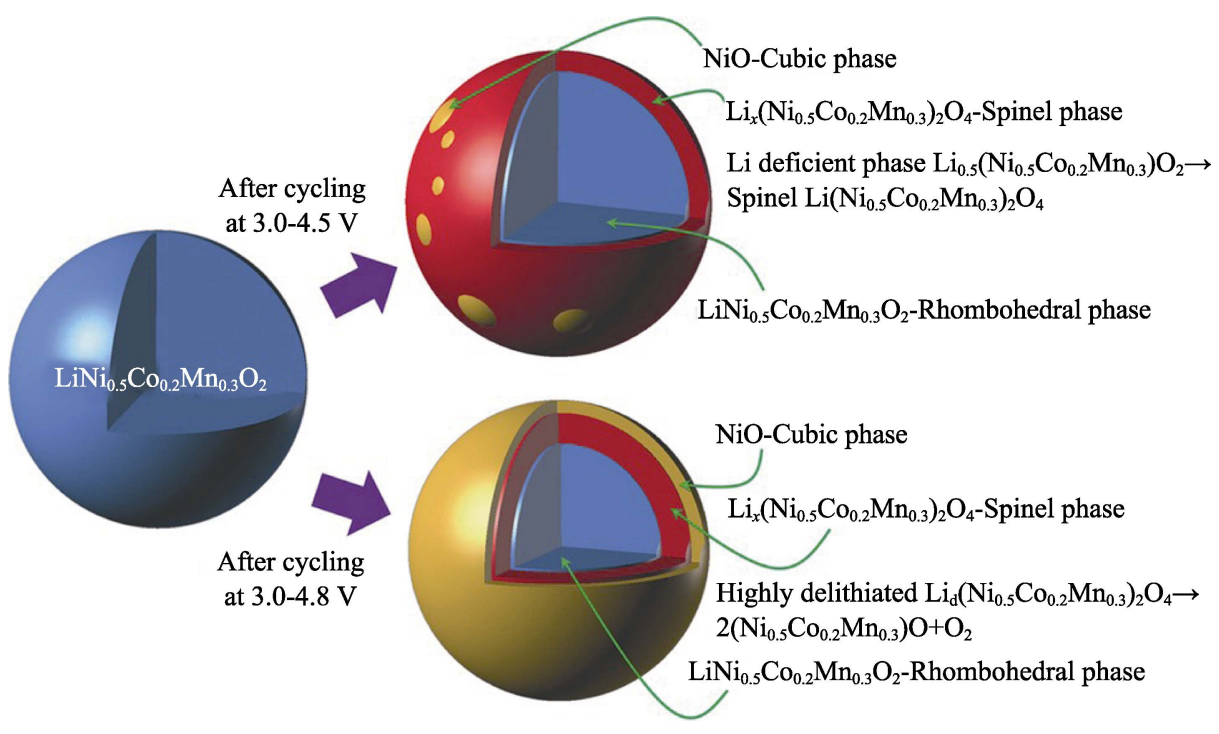

图 2 NCM523 高电压循环过程中的降解机制和相变 ${ }^{[20]}$

Fig. 2 Degradation mechanisms of NCM523 and phase transformation after cycle tests under high-voltage conditions ${ }^{[20]}$

学性能提升机理的综述文章却较少。本文将从这两 个方面系统介绍近年来三元正极材料包覆和掺杂改 性研究的最新进展及其成果。

\section{1 包覆改性}

电极材料与电解液之间的界面是电荷转移、 $\mathrm{Li}^{+}$ 扩散和副反应的关键问题所在, 表面包覆改性是一 种解决界面问题的有效方法。

\subsection{HF 清除剂}

在锂离子电池中, 电解液作为传递锂离子的媒 介, 主要由有机溶剂和电解质盐组成。现阶段商业 化的电解液中, 使用最多的锂盐为 $\mathrm{LiPF}_{6}$ 。有研究表 明，当温度超过 $50{ }^{\circ} \mathrm{C}$ 时，电解液中的 $\mathrm{LiPF}_{6}$ 会发生 分解(式(1) $)^{[24-25]}$ 。在商业化的电解液中, 完全把水 除去是不可能的, 微量水导致 $\mathrm{LiPF}_{6}$ 分解(式 $\left.(2,3)\right)$ 生成 $\mathrm{HF}^{[26]}$ 。 $\mathrm{HF}$ 侵蚀致使正极材料溶解加剧 ${ }^{[13]}$, 溶 解后的金属离子聚集在正负极极片表面。随着电化 学反应的进行，负极表面的金属离子被还原为金属 单质，造成安全隐患。而正极附近的金属离子则有 可能形成 $\mathrm{Ni}-\mathrm{O} 、 \mathrm{Co}-\mathrm{O} 、 \mathrm{Mn}-\mathrm{O}$ 等非电化学活性物 质，不利于电子传输。此外，腐蚀后的正极材料颗粒 与导电剂等物质的接触变差, 引起循环过程中电压 降低和阻抗上升。过渡金属溶解后，正极材料表面 出现岩盐相或尖晶石相, 且相变的种类与正极材料 的组成有关。对于 NCM523, 截止电压较高时 $(4.8 \mathrm{~V})$ 由于失氧, 表面重构主要生成岩盐相, 但是在正常 的循环过程中则主要是尖晶石相 ${ }^{[20]}$ 。 NCM442 恰好 相反, 循环过程中表面主要是岩盐相 ${ }^{[27]}$ 。有趣的是, 尽管 NCM811 的循环性能更差, 但循环过程中基本
观察不到明显的结构变化, 其容量衰减主要来源于 高脱 $\mathrm{Li}$ 表面和电解液之间的副反应 ${ }^{[28]}$ 。

$$
\begin{gathered}
\mathrm{LiPF}_{6} \rightarrow \mathrm{LiF} \downarrow+\mathrm{PF}_{5} \\
\mathrm{PF}_{5}+\mathrm{H}_{2} \mathrm{O} \rightarrow \mathrm{POF}_{3}+2 \mathrm{HF} \\
2 \mathrm{POF}_{3}+3 \mathrm{Li}_{2} \mathrm{O}^{-} \rightarrow 6 \mathrm{LiF} \downarrow+\mathrm{P}_{2} \mathrm{O}_{5} \downarrow\left(\text { or } \mathrm{Li}_{x} \mathrm{POF}_{y}\right)
\end{gathered}
$$

选择合适的包覆剂隔绝正极材料与电解液是一 种常见的保护思路, 常用包覆物质为氧化物。通过 ALD (Atomic Layer Deposition, 原子层沉积) 在 $\mathrm{NCM} 523$ 表面沉积一层 $\mathrm{Al}_{2} \mathrm{O}_{3}$ 之后, 可以显著减少 $\mathrm{NCM} 523$ 与电解液的副反应 ${ }^{[29]} 。 \mathrm{Al}_{2} \mathrm{O}_{3}$ 包覆对 NCM811 的初始性能没有明显影响, 但随着循环周 数的增加, 包覆材料的优越性逐步得到体现 ${ }^{[30]}$ 。这 不仅与 $\mathrm{Al}_{2} \mathrm{O}_{3}$ “保护层”能够隔绝正极材料与电解液 有关，更重要的是改善了高镍材料的表面性质，如 降低表面转移电阻、提高离子导电率等。此外, 包 覆层还能够降低正极材料的吸水性能, 而水含量与 电池的长期性能紧密相关 ${ }^{[31-32]}$ 。其他氧化物如 $\mathrm{SiO}_{2}{ }^{[33]} 、 \mathrm{WO}_{3}{ }^{[34]} 、 \mathrm{Y}_{2} \mathrm{O}_{3}{ }^{[35]} 、 \mathrm{Cr}_{2} \mathrm{O}_{3}{ }^{[36]} 、 \mathrm{Nb}_{2} \mathrm{O}_{5}{ }^{[37]}$ 等 也能改善循环和提高材料在空气中稳定性, 但 $\mathrm{Al}_{2} \mathrm{O}_{3}$ 对材料性能的提升最有效 ${ }^{[37-38]}$ 。

尽管氧化物包覆剂的作用十分明显，但并不能 完全阻止 HF 对正极材料的侵蚀 ${ }^{[16]}$ 。使用 $\mathrm{Al}_{2} \mathrm{O}_{3}$ 包 覆后依然会检测到 Ni-F、Mn-F, 即 HF 与除了与包 覆剂 $\mathrm{Al}_{2} \mathrm{O}_{3}$ 反应之外，同样会与 $\mathrm{NCM}$ 本体反应 ${ }^{[13,16]}$ 。 这些副产物附着在正极的表面, 增加循环过程中的 电阻。此外, 包覆效果与包覆方法及包覆层厚度紧 密相关 ${ }^{[39-41]}$, 虽然包覆效果可以通过新型包覆手段 (如 ALD) 实现, 但目前这些新型方法还难以大规模 地商业化使用。再者，尽管在这个过程中 HF 被消耗 后形成更稳定的氟化物，可以阻止其对正极材料的 
进一步侵蚀。但氧化物与 $\mathrm{HF}$ 的反应产物中有 $\mathrm{H}_{2} \mathrm{O}$, 会加剧电解液中 HF 的产生。

由于 $\mathrm{AlF}_{3}$ 对 $\mathrm{HF}$ 更稳定, 且反应产物中没有 $\mathrm{H}_{2} \mathrm{O}$, 同时 $\mathrm{F}$ 的电负性高, 与正极材料表面氧的键 合更强，可以进一步抑制表面副反应，因此是一个 更好的选择 ${ }^{[42-43]}$ 。包覆 $\mathrm{AlF}_{3}$ 后, NCM523 的晶格参 数没有改变, 说明 $\mathrm{AlF}_{3}$ 并没有进入晶格, 而仅仅是 包覆在 NCM 表面 ${ }^{[44]}$ 。由于循环过程中没有额外产 生 $\mathrm{H}_{2} \mathrm{O}$, 因此 $\mathrm{AlF}_{3}$ 包覆层可以减缓 $\mathrm{LiPF}_{6}$ 的分解和 $\mathrm{Mn}$ 的溶解, 即可以增强材料的热稳定性和循环稳 定性, 尤其是在脱锂量较大的情况。更重要的是, 经 过 60 周循环后裸料的电荷转移阻抗增加了约 25 倍, 而包覆样品仅增加了约 2.7 倍 ${ }^{[44]}$ 。 $\mathrm{CeF}_{3}$ 在高温下呈 现良好的电化学惰性, 在酸性环境下具有很好的稳 定性 ${ }^{[45]}$, 并且室温时离子电导率很高 ${ }^{[46-47]}$, 符合包 覆材料的各项要求。通过湿法工艺在 NCM111 表面 沉积一层 $\mathrm{CeF}_{3}$ 后 ${ }^{[48]}$, 能够有效防止 HF 对活性材料 表面的持续侵蚀、延迟表面钝化层的生长; 同时还 能起到稳定结构的作用, 提升材料的高电压(2.5 $4.5 \mathrm{~V})$ 倍率性能和循环性能。不同的氟化物阳离子价 态可以影响其包覆效果。例如, $\mathrm{MgF}_{2}$ 和 $\mathrm{LaF}_{3}$ 均能提 升 NCM523 的倍率和循环性能 ${ }^{[49]}$, 对高电压(3.0 $4.8 \mathrm{~V}$ )循环过程中稳定晶体结构也有积极作用, 但 $\mathrm{LaF}_{3}$ 包覆材料的高倍率性能 (6C) 更优(图 3), 而 $\mathrm{MgF}_{2}$ 包覆更有利于提升热稳定性和高电压循环性 能。两种包覆材料 50 周充放电循环后的 XRD(X-ray Diffraction, $X$ 射线衍射) 图谱也有所不同, $\mathrm{LaF}_{3}$ 包覆 材料的(015)衍射峰强度明显降低, $\mathrm{MgF}_{2}$ 包覆材料的 (110)衍射峰则出现轻微䢃裂。这可能与氟化物的热 力学稳定性有关, 详细的机理还有待进一步研究 ${ }^{[00]}$ 。

\section{2 残碱清除剂}

研究结果显示, 残碱对材料的使用和性能发挥 均有不利影响, 因此需要使用合适的方法将材料表 面的残碱控制在一定的范围内。水洗是一种降低残 碱的有效处理方式，但水洗后材料的放电比容量明 显降低，存储稳定性变差，阳离子混排也更严重。有 研究 ${ }^{[9]}$ 显示, 在空气中存储 $30 \mathrm{~d}$ 后水洗样品的总碱 量是未水洗样品的 2 倍多。包覆除了可以消耗 HF 之外，也是一种有效降低残碱的方法 ${ }^{[51]}$ 。

$\mathrm{H}_{2} \mathrm{SiO}_{3}$ 与 $\mathrm{NCM}$ 表面残余的 $\mathrm{LiOH} / \mathrm{Li}_{2} \mathrm{CO}_{3}$ 反应, 降低残碱的同时形成一层致密的 $\mathrm{Li}_{2} \mathrm{Si}_{2} \mathrm{O}_{5}$ 包覆层 ${ }^{[52]}$ 。 当 $\mathrm{H}_{2} \mathrm{SiO}_{3}$ 的添加量为 $1 \mathrm{wt} \%$ 时，总碱量由 $0.46 \%$ 降 低到 $0.19 \%$, 效果十分明显。消耗残碱有效改善了 材料表面性能, 有利于提升放电容量和库仑效率; 而 $\mathrm{Li}_{2} \mathrm{Si}_{2} \mathrm{O}_{5}$ 则提高了离子电导率、降低了极化, 有 利于提升倍率性能。但是当添加量增加至 $2 \mathrm{wt} \%$ 时,
由于过量的 $\mathrm{H}_{2} \mathrm{SiO}_{3}$ 分解形成惰性 $\mathrm{SiO}_{2}$, 阻碍了 $\mathrm{Li}$ 离子脱嵌, 包覆效果反而有所下降(表 1)。值得注意 的是, 采取这种思路进行包覆处理时, 对正极材料 的保护和性能提升往往是多维度的。 $\mathrm{H}_{3} \mathrm{PO}_{4}$ 与残碱 反应生成 $\mathrm{Li}_{3} \mathrm{PO}_{4}$ (图 4), 除降低残碱提高离子电导 率外, 还能吸收电解液中的 $\mathrm{H}_{2} \mathrm{O}$ 和 $\mathrm{HF}$, 减少活性物 质与电解液的副反应, 改善材料的循环性能 ${ }^{[53-55]}$ 。
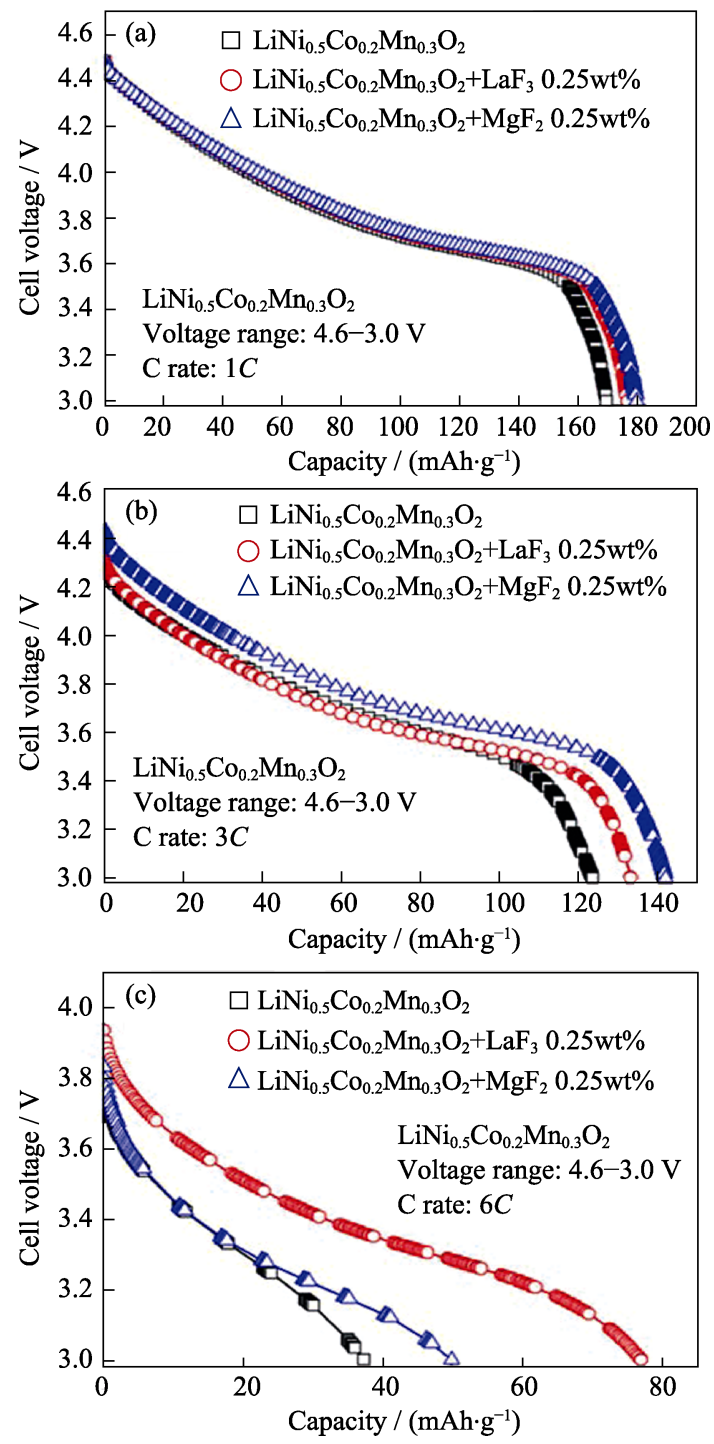

图 3 NCM523 裸样和包覆样的第 5 周放电曲线 $(3.0 \sim 4.6 \mathrm{~V})^{[49]}$ Fig. 3 th discharge profiles of pristine and coated NCM523 $(3.0 \sim 4.6 \mathrm{~V})^{[49]}$

(a) $1 C$; (b) $3 C$; (c) $6 C$

\section{表 1 不同包覆量时锂离子电池的放电比容量 ${ }^{[52]}$ \\ Table 1 Discharge capacities of Li-ion batteries with different coating amounts ${ }^{[52]}$}

\begin{tabular}{|c|c|c|c|c|c|c|}
\hline \multirow[b]{2}{*}{ Sample } & \multicolumn{6}{|c|}{ Discharge capacity $/\left(\mathrm{mAh} \cdot \mathrm{g}^{-1}\right)$} \\
\hline & $0.1 C$ & $1 C$ & $2 C$ & $5 C$ & $10 C$ & $0.1 C$ \\
\hline & \multicolumn{6}{|c|}{$/ 3$ cycles $/ 5$ cycles $/ 5$ cycles $/ 5$ cycles $/ 5$ cycles $/ 5$ cycles } \\
\hline 0 & 196.8 & 168.9 & 155.8 & 131.8 & 94.5 & 184.6 \\
\hline $1 \mathrm{wt} \%$ & 213.9 & 185.5 & 170.2 & 148.1 & 121.6 & 207.2 \\
\hline $2 w t \%$ & 203.8 & 161.1 & 147.9 & 122.6 & 92.1 & 195.7 \\
\hline
\end{tabular}

* After cycling at $10 \mathrm{C}$ 
(a)

(b)

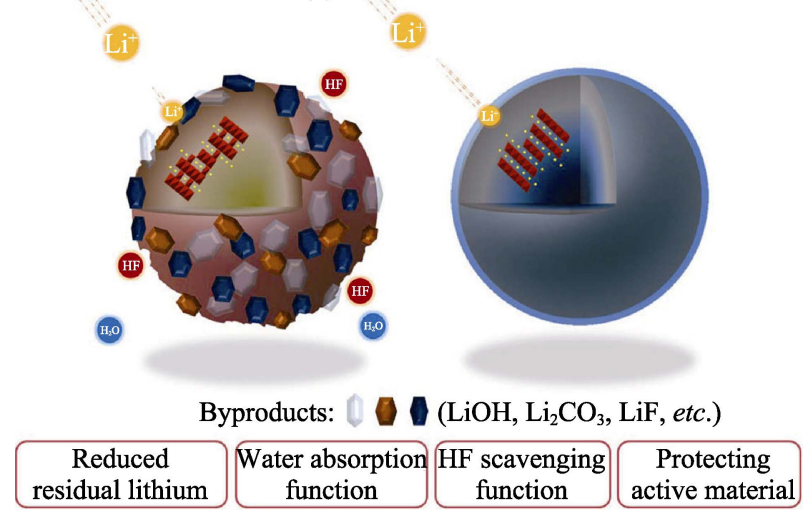

图 4 循环 150 次后(a)裸样和(b) $\mathrm{Li}_{3} \mathrm{PO}_{4}$ 包覆的 NCM622 $(1 \mathrm{wt} \%)$ 的表面副产物示意图 ${ }^{[53]}$

Fig. 4 Schematic illustration of byproducts on the surfaces of (a) bare and (b) lithium phosphate-coated NCM622 after cycling 150 times $^{[53]}$

此种思路可以扩展至其他类型的正极材料, 如可利 用 $\left(\mathrm{NH}_{4}\right)_{2} \mathrm{SO}_{4}$ 对固溶体材料进行表面处理, 形成 $\mathrm{Li}_{2} \mathrm{SO}_{4}$ 包覆层 ${ }^{[56]}$ 。使用氧化物进行包覆时, 虽然氧 化物主要起“保护层”的作用，但文献[57]报道显示， 当热处理温度合适时，氧化物包覆剂也可能与正极 材料表面的残碱发生反应。

除无机化合物外，有机物也可以用于正极材料 的包覆。 $\mathrm{Xu}$ 等 ${ }^{[58]}$ 使用质子化聚苯胺(PANI)的 NMP (1-methyl-2-pyrrolidinone, 1-甲基-2-吡咯烷酮)溶液 对 NCM811 进行洗涤, PANI 中的 $\mathrm{H}^{+}$可以与残碱反 应、降低总碱量，同时由于 PANI 在 NMP 中是弱酸, 因此不会与 NCM 本体反应。残碱减少和 PANI 包覆 的双重作用有利于改善材料的倍率、循环和高温性 能。石墨烯具有优异的电学和热学性能, 在锂离子 电池中是一种理想的添加剂 ${ }^{[59]}$, 但缺点也很明显: 其二维平面结构很难与活性材料表面紧密结合, 在 充放电过程中容易脱落。使用环氧官能化硅烷 (KH650)和还原氧化石墨烯(RGO)对 NCM811 进行 双包覆 ${ }^{[60], K H 650 ~}$ 同时起到消耗- $\mathrm{OH}$ 、降低残碱和 固化 RGO 的作用, 两者结合可以促进电子的传递, 并在循环过程中保护活性物质不受侵蚀，降低初始 不可逆容量损失, 减少电解质与电极的副反应。同 理, 以聚乙烯吡咯烷酮(PVP)诱导剂在 NCM811 表 面包覆一层聚苯胺, 也可达到相同的效果 ${ }^{[61]}$ 。正极 材料通常是由一次颗粒堆积形成的球形二次颗粒, 仅在二次颗粒表面包覆, 对材料内部导电率的提升 有限。借助球磨、喷雾干燥工艺, 将碳材料包覆在 一次颗粒表面并填充在一次颗粒之间，可形成更优 良的导电网络，从而更好地改善正极材料的倍率性 能和循环性能 ${ }^{[62-64]}$ 。
传统的包覆方式通常是在材料生产过程中完成, 最近有研究组 ${ }^{[65]}$ 采用新方法, 将包覆过程与电池制 备时的匀浆过程相结合，取得了不错的效果。正极 匀浆时加入一定量的 $\mathrm{S}$ 将 $\mathrm{Ni}^{3+}$ 还原为 $\mathrm{Ni}^{2+}, \mathrm{S}$ 被氧化 为 $\mathrm{Li}_{2} \mathrm{~S}_{2} \mathrm{O}_{3}$, 并在首次充电时继续氧化形成 $\mathrm{Li}_{2} \mathrm{SO}_{4}$ 。 其作用机理如式(4 8)所示。这种处理方式对材料的 循环性能没有明显影响，但是可以显著提升倍率性 能。类似地, 在匀浆过程中加入 $\mathrm{LiPF}_{6}$ 也可以起到相 同的效果，反应产物为更稳定的 $\mathrm{Li}_{3} \mathrm{PO}_{4}$ 和 $\mathrm{LiF}$ ，提 升材料循环性能的同时还可以降低表面电阻, 提升 材料的倍率性能 ${ }^{[66]}$ 。

$$
\begin{gathered}
4 \mathrm{LiMO}_{2}+2 \mathrm{~S} \rightarrow 4 \mathrm{MO}+\mathrm{Li}_{2} \mathrm{~S}_{2} \mathrm{O}_{3}+\mathrm{Li}_{2} \mathrm{O} \\
\mathrm{Li}_{2} \mathrm{~S}_{2} \mathrm{O}_{3}+5 \mathrm{Li}_{2} \mathrm{O}-8 \mathrm{e} \rightarrow 2 \mathrm{Li}_{2} \mathrm{SO}_{4}+8 \mathrm{Li}^{+} \\
\mathrm{Li}_{2} \mathrm{~S}_{2} \mathrm{O}_{3}+5 \mathrm{Li}_{2} \mathrm{CO}_{3}-8 \mathrm{e} \rightarrow 2 \mathrm{Li}_{2} \mathrm{SO}_{4}+5 \mathrm{CO}_{2}+8 \mathrm{Li}^{+} \\
2 \mathrm{LiMO}_{2}+\mathrm{S}+2 \mathrm{Li}_{2} \mathrm{O}-4 \mathrm{e} \rightarrow 2 \mathrm{MO}^{+} \mathrm{Li}_{2} \mathrm{SO}_{4}+4 \mathrm{Li}^{+} \\
2 \mathrm{LiMO}_{2}+\mathrm{S}+2 \mathrm{Li}_{2} \mathrm{CO}_{3}-4 \mathrm{e}^{+} \\
2 \mathrm{MO}+\mathrm{Li}_{2} \mathrm{SO}_{4}+2 \mathrm{CO}_{2}+4 \mathrm{Li}^{+}
\end{gathered}
$$

\section{3 结构稳定剂}

高镍三元正极材料循环性能不佳，除了副反应 之外, 还与充放电过程中的表面结构变化和二次颗 粒破碎有关 ${ }^{[27,67-68]}$ 。某些特定的包覆剂在隔绝电解 液、减少副反应的同时, 也能起到稳定表面结构和 抑制微裂纹的作用。如硅酸盐和磷酸盐, 较强的 $\mathrm{Si}=\mathrm{O} 、 \mathrm{P}=\mathrm{O}$ 键使过渡金属与 $\mathrm{SiO}_{3} 、 \mathrm{PO}_{4}$ 聚阴离子间 的共价键更强; 同时, 硅酸盐/磷酸盐比金属氧化物 具有更高的热力学与电化学稳定性, 因此能够提升 正极材料结构稳定性，提升高电压或高温性能。 $\mathrm{Li}_{2} \mathrm{SiO}_{3}$ 包覆层能有效地隔绝高电压下电极一电解液 界面上的副反应, 更重要的是, 当锂离子在高压下 发生深度脱层时，包覆层增强了正极材料的结构稳 定性 ${ }^{[69]}$ 。此外, $\mathrm{Li}_{2} \mathrm{SiO}_{3}$ 还可以增强锂离子在电极/ 电解质界面的扩散，防止正极材料颗粒在循环过程 中的粉化 ${ }^{[70]}$ 。 $\mathrm{Li}_{3} \mathrm{PO}_{4}$ 在提升材料的倍率性能的同时, 又可以阻止 $\mathrm{HF}$ 和 $\mathrm{POF}_{3}$ 的侵蚀，提升材料的循环性 能 ${ }^{[71]}$ 。有证据表明, $\mathrm{Li}_{3} \mathrm{PO}_{4}$ 还能够减缓高温下的相 转变, 正极材料的初始热分解温度和放热量均明显 下降, 相转变温度提升, 并且可以抑制尖晶石和岩 盐结构之间的相转变。 $\mathrm{LiFePO}_{4}(\mathrm{LFP})$ 正极材料得益 于其无机性质和化学稳定性, 具有优异的循环性能, 作为包覆剂使用时能够提升三元材料的高温循环稳 定性，同时又不会降低首周放电比容量 ${ }^{[72]}$ 。但是, LFP 的导电性较差, 需要通过工艺严格控制颗粒大 小和包覆层厚度 ${ }^{[73]}$ 。为尽量提高三元材料的放电容 量, 需要提升其充放电截止电压, 但高电压下副反 应和不可逆相变加剧, 影响循环性能和安全性能。 在以 LFP 包覆的 NCM523 为正极组装的 5 Ah 软包 
电池中, LFP 包覆层抑制了层状一尖晶石一岩盐相 的相转变, 因此该电池能够耐受 $4.5 \mathrm{~V}$ 的高电压, 且 在满电状态下不会发生热失控 ${ }^{[74-75]}$, 其他磷酸盐也 有类似报道 ${ }^{[76-77]}$ 。自从索尼公司首次推出 $\mathrm{LiCoO}_{2} / \mathrm{C}$ 二次电池以来, $\mathrm{LiCoO}_{2}$ 作为正极材料取得了巨大的 成功。有鉴于此, 研究者将 $\mathrm{LiCoO}_{2}$ 用于三元材料的 包覆, 发现其可以减少三元材料表面的 $\mathrm{NiO}$ 结构, 提升电化学性能 ${ }^{[78-79]}$ 。此外, 由于 $\mathrm{LiCoO}_{2}$ 的结构稳 定性, 同时还能提升三元材料在空气中的存储性能, 减少表面镍价态、水分、残碱含量以及降低电化学 性能受暴露时间的影响 ${ }^{800}$ 。

除材料本身的结构稳定性之外, 循环过程中二 次颗粒内部产生的微裂纹也是导致正极材料循环性 能下降的原因之一: 产生微裂纹后, 电解液即会浸 入, 并在颗粒内发生不可逆分解, 分解产物沿晶间 裂纹的积累加速了容量衰减(图 5) ${ }^{[81]}$ 。在 $\mathrm{Li}_{2} \mathrm{SiO}_{3}$ 包 覆的 NCM523 中, 富硅区不仅集中在颗粒的表面, 在晶界处、甚至在 $400 \mathrm{~nm}$ 深处也有 $\mathrm{Si}$ 元素分布 ${ }^{[82]}$ 。 富硅区抑制了裂纹的形成，阻止了电解质溶液在颗 粒内的侵入和分解。 $\mathrm{LBO}$ (硼酸锂)材料具有良好的 离子电导率和高氧化稳定性, 更重要的是在提升三

(a)

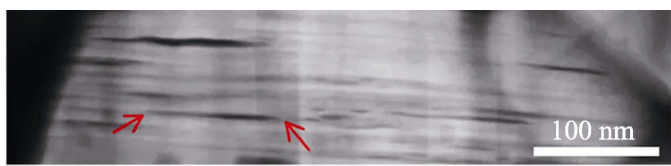

(b)

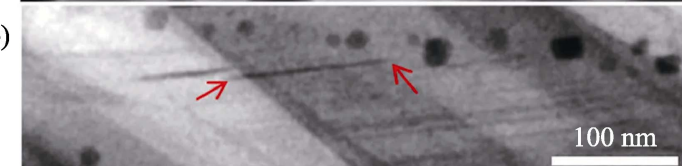

(c)

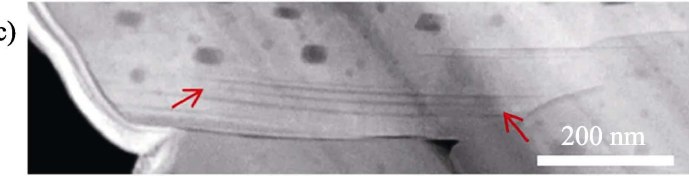

(d)

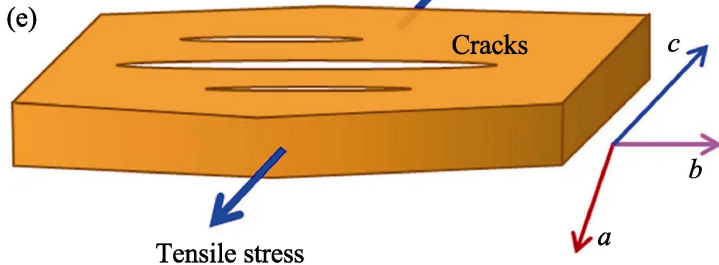

图 5100 周循环 $(4.7 \mathrm{~V})$ 后正极材料颗粒内部微裂纹的 (a d)SEM 照片及其(e)形成示意图 ${ }^{[81]}$

Fig. 5 (a-d) SEM images of the cathode cracks in the particles cycled 100 times $(4.7 \mathrm{~V})$, and (e) schematic diagram showing crack formation $^{[81]}$
元材料的高电压性能的同时 ${ }^{[83]}$, 也能够抑制循环过 程中微裂纹的产生，将 NCM811 在 $4.6 \mathrm{~V}$ 循环时微 裂纹的出现周数由 106 周延长至 211 周 ${ }^{[84]}$ 。

\section{4 小结}

由于在活性材料表面引入了其他物质，并且绝 大多数包覆物不具有电化学活性, 因此尽管包覆改 性能够提升材料的性能，但往往也会带来新的问 题。比如, 传统的包覆剂如 $\mathrm{Al}_{2} \mathrm{O}_{3} 、 \mathrm{SiO}_{2} 、 \mathrm{TiO}_{2}$ 、 $\mathrm{AlPO}_{4} 、 \mathrm{CoPO}_{4}$ 等离子电子传导率低, 容易导致包覆 后材料的极化增加、容量降低、倍率降低等, 而选 用快离子导体则可避免相应问题 ${ }^{[54,69-71,85]}$ 。 $\mathrm{Zr}$ 对提 升循环保持率有益处，但对初始容量和残碱作用不 明显, $P$ 则相反。使用 $\mathrm{Zr} / \mathrm{P}$ 双包覆, 借助 $\mathrm{Zr}$ 和 $\mathrm{P}$ 的 协同作用，可以同时提升样品的初始容量和循环性 能, 残碱的降低也较为可观 ${ }^{[86]}$ 。包覆剂中的高价态 金属离子 $\left(\right.$ 如 $\mathrm{Ti}^{4+}$ )容易进入晶格, 加剧 $\mathrm{Li} / \mathrm{Ni}$ 混排, 使用纳米 $\mathrm{Li}_{2} \mathrm{TiO}_{3}$ 进行包覆则可避免 ${ }^{[87]} 。 \mathrm{Cr}_{8} \mathrm{O}_{21}$ 包 覆 NCM523 的结果表明, 虽然包覆量为 $2.5 \mathrm{wt} \%$ 时不 可逆容量有所增加 $(15.6 \mathrm{mAh} / \mathrm{g})$, 但却拥有最优的 循环性能 ${ }^{[88]}$ 。三元正极材料首次库伦效率普遍不高, 这与循环初期氧空位的形成有关 ${ }^{[89]}$, 包覆改性能够 限制氧空位产生，降低首周不可逆容量。Kalaiselvi 课题组 ${ }^{[90]}$ 的研究结果显示, 在 $\mathrm{Al}_{2} \mathrm{O}_{3} 、 \mathrm{Bi}_{2} \mathrm{O}_{3} 、 \mathrm{In}_{2} \mathrm{O}_{3}$ 三种包覆物中, $\mathrm{Bi}_{2} \mathrm{O}_{3}$ 包覆料的不可逆容量最低, 同 时也具有最好的结构稳定性, 甚至可以耐受 $5.2 \mathrm{~V}$ 的高电压。

综上，包覆剂的物化性质、包覆量、包覆方式、 混合方式等对正极材料性能的影响是多方面的、复 杂的, 且在改善某一方面性能的同时, 经常需要以 牺牲其他性能为代价。而通过对包覆剂的选择和包 覆工艺的优化, 则可减少负面作用。这就要求研究 者综合考虑, 开展横向(如: 不同类型包覆剂)和纵 向(如: 相同类型不同阳离子价态包覆剂)的对比研 究，全面提升 NCM 材料的性能。

\section{2 掺杂}

包覆对解决材料表面问题效果显著, 但对晶体 结构的作用却十分有限，而体相结构也是影响材料 性能的关键所在, 因此需要借助掺杂改性进一步提 升材料的性能。

\section{1 减少不稳定元素}

三元材料中普遍存在锂镍混排现象, 造成 $\mathrm{Li}-\mathrm{O}$ 层间距减小和扩散阻力增大。锂位的过渡金属离子 也会阻碍锂离子传导，导致锂离子扩散能垒增高, 
倍率性能下降。文献[91]报道了多种控制锂镍混排 的方法, 由于锂镍混排不仅发生在材料制备阶段, 也发生在电池的循环过程中, 因此除了需要严格控 制高温合成条件之外, 体相掺杂也是一种必要的手 段, 可以有效地改善高镍三元材料的电化学性能。

由于离子半径相当 $\left(\mathrm{Li}^{+}=0.076 \mathrm{~nm}, \mathrm{Mg}^{2+}=0.072 \mathrm{~nm}\right.$, $\left.\mathrm{Ni}^{2+}=0.069 \mathrm{~nm}\right)$, 当掺杂量 $<2 \mathrm{wt} \%$ 时, $\mathrm{Mg}^{2+}$ 主要占据 $\mathrm{Li}$ 位 ${ }^{[92]}$, 在脱锂量逐渐增加、电压逐渐增高的过程 中, $\mathrm{Mg}^{2+}$ 的支柱作用能够减轻各向异性的晶格变 化 ${ }^{[93]}$ 。较小的 $c$ 轴变化率和稳定的电压平台均表明 掺杂样具有良好的结构可逆性 ${ }^{[94]}$ 。值得注意的是, $\mathrm{Mg}$ 掺杂进入 $\mathrm{Li}$ 层会导致 $\mathrm{Li} / \mathrm{Ni}$ 混排加剧 $(1.8 \% \rightarrow$ $2.3 \%)$, 但对倍率性能影响不大, 且 200 周循环后掺 杂样的 $\mathrm{Li} / \mathrm{Ni}$ 混排则明显小于裸样 $(4.0 \% \rightarrow 7.8 \%)$ 。当 脱锂量较大时, 相邻过渡金属层间 $\mathrm{O}^{2-}-\mathrm{O}^{2-}$ 的强斥 力导致层状结构坞塌和不可逆相变, 造成 $\mathrm{NCM}$ 高 电压循环性能不佳。 $\mathrm{Mg}^{2+}$ 掺杂可以降低 $\mathrm{O}^{2-}-\mathrm{O}^{2-}$ 之 间的斥力、提高 $\mathrm{O}-\mathrm{M}-\mathrm{O}$ 的键能, 增强层状结构的稳 定性，有效抑制充放电过程中的相转变 ${ }^{[95]}$ 。Aurbach 课题组 ${ }^{[96]}$ 研究了 $\mathrm{Mo}^{6+}$ 掺杂的 NCM523, 认为 $\mathrm{Mo}^{6+}$ 与 $\mathrm{Mg}^{2+}$ 不同, 倾向于在表面富集, 并且在晶格中处 于 $\mathrm{Ni}$ 位。掺杂对样品的放电比容量和循环性能均有 有益影响: $1 C \sim 4 C$ 倍率放电比容量提升 $20 \% \sim 30 \%$; 截止电压在 $4.3 \sim 4.6 \mathrm{~V}$ 范围内时常温 $\left(30{ }^{\circ} \mathrm{C}\right)$ 和高温 $\left(45{ }^{\circ} \mathrm{C}\right)$ 循环容量衰减率均有所减缓(图 6)。

除取代金属离子外，也可进行阴离子 $(\mathrm{O})$ 掺杂 ${ }^{[97]}$ 。 第一性原理计算和中子粉末衍射(NPD)表明 ${ }^{[98], F}$ 原 子取代 $\mathrm{O}$ 原子可以促使相邻的 $\mathrm{Li} 、 \mathrm{Ni}$ 互换位置, 诱 导形成稳定的局部卤化物八面体(LOSH)。NPD 和 $\mathrm{XRD}$ 结果显示, 通过改变 $\mathrm{F}$ 掺杂量可以调整这种 “反位”缺陷的比例, 而缺陷比例与材料的性能密切 相关。拥有 $5.7 \%$ 缺陷的掺杂样具有良好的高温循环 性能 $\left(55{ }^{\circ} \mathrm{C}\right)$ 和倍率性能, 原因是 LOSH 使 $c$ 轴增大, 更有利于 $\mathrm{Li}$ 离子的迁移。缺陷还可以提高材料的热 稳定性, $5.7 \%$ 缺陷可以将热分解温度由 $179.51{ }^{\circ} \mathrm{C}$ 提 高到 $226.96{ }^{\circ} \mathrm{C}$ 。可能原因有 2 个: 1) Ni 迁移到 $\mathrm{Li}$ 层诱导 LOSH 的产生; 2)反位缺陷反过来阻止更多 的 $\mathrm{Ni}$ 迁移到 $\mathrm{Li}$ 层, 抑制层状结构的相变。 $\mathrm{Li}$ 等 ${ }^{[99]}$ 通过对 NCA 进行氟掺杂改性, 也得到类似结论。由 于 $\mathrm{Li}-\mathrm{F}$ 键强于 $\mathrm{Li}-\mathrm{O}$ 键, 氟会部分替代氧的位置。 因此在氟掺杂之后, 材料表面的 $\mathrm{Ni}^{2+}$ 数量有所增加, 而活性的 $\mathrm{Ni}^{3+}$ 数量有所减少, 加剧锂镍混排, 首次 放电比容量有所下降; 但是材料表面的活性 $\mathrm{Ni}^{3+}$ 减 少, 有利于减少界面副反应, 抑制极化以及避免循 环过程中阻抗升高, 提升材料的循环性能、倍率性 能、高温性能和过充性能等。
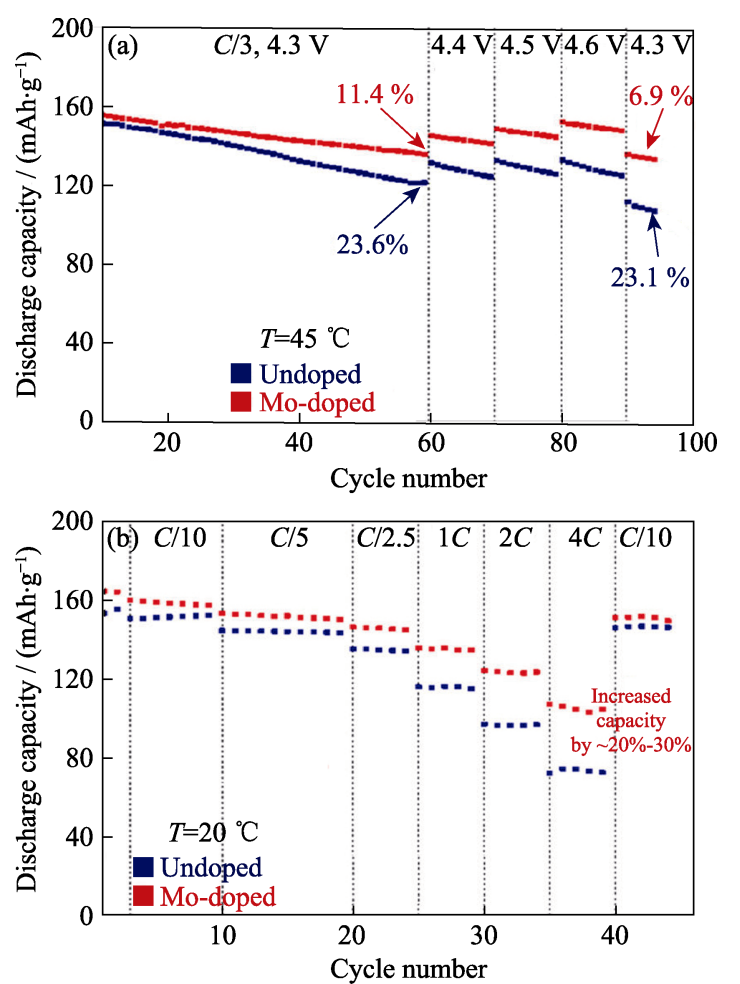

图 6 在(a) $45{ }^{\circ} \mathrm{C} \quad(4.3 、 4.4 、 4.5 、 4.6 \mathrm{~V}, C / 3)$ 和(b) $30{ }^{\circ} \mathrm{C}$ (2.8 4.3 V, 不同倍率)条件下 NCM523 裸样和 Mo 掺杂样的 循环曲线 ${ }^{[96]}$

Fig. 6 Typical cycling performance of undoped and Mo-doped NCM523 at (a) $45{ }^{\circ} \mathrm{C}$ (4.3, 4.4, 4.5, and $4.6 \mathrm{~V}, \mathrm{C} / 3$ rate) and (b) $30{ }^{\circ} \mathrm{C} \quad(2.8-4.3 \mathrm{~V} \text {, different rates })^{[96]}$

\section{2 降低 $\mathrm{Li} / \mathrm{Ni}$ 混排}

锂镍混排对 NCM 材料的电化学性能和结构稳 定性均有不利影响, 有必要进行控制。但如 2.1 中所 言, 某些掺杂元素(如 $\mathrm{Mg}$ )会加剧 $\mathrm{Li} / \mathrm{Ni}$ 混排, 因此 对材料进行掺杂改性提高结构稳定性的同时, 还需 要考虑对混排的影响。

$\mathrm{Nb}$ 掺杂主要以 +5 价形式占据 NCM111 的 $3 b$ 位 置 ${ }^{[100]}$, 可以有效减少阳离子混排。当掺杂量为 $2 \mathrm{wt} \%$ 时, $\mathrm{Ni}_{3 \mathrm{a}} /\left(\mathrm{Ni}_{3 \mathrm{a}}+\mathrm{Li}_{3 \mathrm{a}}\right)$ 由裸样的 $5.73 \%$ 减少到 $3.13 \%$, 相 应的 $0.1 C$ 放电比容量 $(2.7 \sim 4.3 \mathrm{~V})$ 由 $151.9 \mathrm{mAh} / \mathrm{g}$ 提 高到 $200.4 \mathrm{mAh} / \mathrm{g}$ 。掺杂后 $c / a$ 值更高, 更有利于锂 离子的脱嵌, 有助于提升倍率性能。由于 $\mathrm{Nb}-\mathrm{O}$ 的 键能大于 $\mathrm{M}-\mathrm{O}(\mathrm{M}=\mathrm{Ni} 、 \mathrm{Co} 、 \mathrm{Mn})$, 因此在循环过程 中结构更稳定。同时为保证价态平衡, 部分 $\mathrm{Mn}^{4+}$ 被 还原形成局部富 $\mathrm{Mn}^{3+}$ 区域, 可以起到增加电导率、 稳定循环的作用。掺杂量是一个很重要的参数, $1 \mathrm{wt} \%$ 的 $\mathrm{Nb}$ 掺杂有助于抑制循环过程中的相转变、 降低电化学极化 ${ }^{[101]}$; 当掺杂量超过 $2 \mathrm{wt} \%$ 时, $\mathrm{Nb}^{5+}$ 不会完全进入晶格, 而是在表面形成 $\mathrm{Nb}_{2} \mathrm{O}_{5}$ 杂相, 导致材料的循环性能下降 ${ }^{[102]}$ 。除影响 $\mathrm{Li} / \mathrm{Ni}$ 混排外, $\mathrm{Ni}^{2+}$ 的迁移还是导致层状结构到尖晶石结构相转变 
的原因 ${ }^{[20,103]}$ 。 $\mathrm{Zr}^{4+}$ 离子的强静电作用使 $\mathrm{Ni}^{2+}$ 迁移阻 力增加，可以抑制层状结构到尖晶石结构的相变 ${ }^{[104]}$ (图 7)。同时 Jahn-Teller 活性 $\mathrm{Ni}^{3+}$ 离子的减少, 也能 够提高结构稳定性和循环稳定性(表 2)。另一方面, 更多的 $\mathrm{Ni}^{2+}$ 被氧化为 $\mathrm{Ni}^{3+}$, 降低了锂镍混排 ${ }^{[105]} \mathrm{Zr}$ 掺杂 NCM523 中活性氧(指 $\mathrm{Li}_{2} \mathrm{CO}_{3} / \mathrm{LiOH}$ 中的氧)的 含量有所减少而晶格氧含量有所增加, 说明 $\mathrm{Zr}$ 进入 了晶格 ${ }^{[106]}$ 。表面的 $\mathrm{Ni}^{3+} / \mathrm{Ni}^{2+}$ 比例增加, 说明锂镍混 排降低，结构稳定性有所增强。当再加入 $\mathrm{Ti}$ 时, $I_{(003)} / I_{(004)}$ 比值进一步升高 $(1.25 \rightarrow 1.31)$, 说明 $\mathrm{Zr} / \mathrm{Ti}$ 双掺杂存在协同作用。单独掺杂 $\mathrm{Ti}$ 可以将 $\mathrm{NCM} 811$ 的 $I_{(003)} / I_{(004)}$ 由 1.35 增加到 1.40 左右, 并且随着掺杂 量的增加, $\mathrm{Li} / \mathrm{Ni}$ 混排逐渐降低 ${ }^{[107]}$ 。掺杂后更完美的 晶体结构提升了材料的高电压倍率性能和循环性能, 原因是 $\mathrm{Ti}$ 增大了晶胞参数, 有利于 $\mathrm{Li}^{+}$的脱嵌。而 充放电过程中的体积变化率变小, 能够减少循环过 程中二次粒子的破碎。但当掺杂量过多时材料的放 电比容量有所降低，这一方面是由于 $\mathrm{Ti}$ 不具有电化 学活性, 另一方面是因为掺杂 $\mathrm{Ti}$ 增加了充放电过程 中的极化。

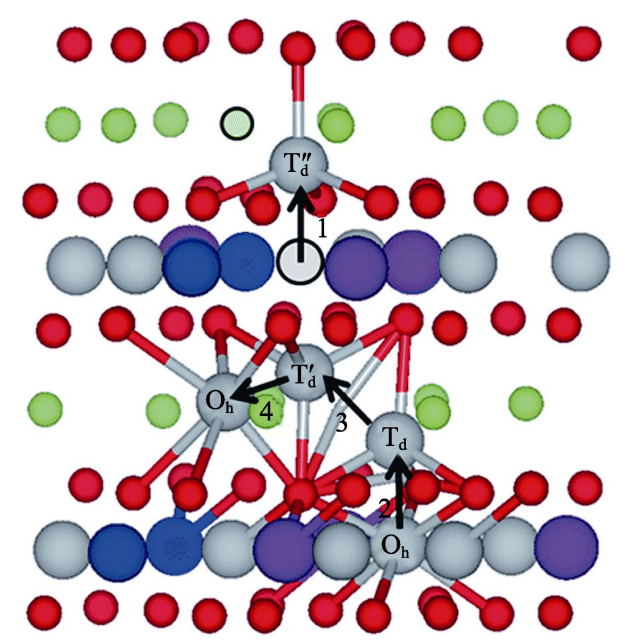

图 7 可能的 $\mathrm{Ni}^{2+}$ 迁移形成尖晶石相机制 ${ }^{[104]}$

Fig. 7 Suggested mechanism for $\mathrm{Ni}^{2+}$ migration leading to a partial spinel nucleus ${ }^{[104]}$

Red: oxygen atoms; Grey: Ni atoms; Violet: Mn atoms; Blue: cobalt atoms

表 2 不同物质掺杂 NCM622 的循环和倍率性能 ${ }^{[104]}$

Table 2 Cycling performances and rate capabilities of NCM622 with different doping agents ${ }^{[104]}$

\begin{tabular}{ccccc}
\hline \multirow{2}{*}{ Sample } & \multicolumn{2}{c}{ Cycling performance $\left(1 C @ 200^{*}\right)$} & & Rate performance \\
\cline { 2 - 3 } \cline { 5 - 5 } & $3.0-4.4 \mathrm{~V}$ & $3.0-4.6 \mathrm{~V}$ & & $16 C / 0.5 C$ \\
\hline Pristine & $75.59 \%$ & $72.99 \%$ & & $64.94 \%$ \\
$\mathrm{Zr}$ & $87.61 \%$ & $81.05 \%$ & & $73.94 \%$ \\
$\mathrm{Zr} / \mathrm{Ti}$ & $94.20 \%$ & $91.71 \%$ & & $79.57 \%$ \\
\hline
\end{tabular}

* 200 cycles at $1 C$ rate

\section{3 稳定氧原子}

充放电过程中高镍材料结构不稳定 ${ }^{[108]}$, 容易 发生层状相一尖晶石相-岩盐相的相转变, 期间伴 随氧气的释放 ${ }^{[109-110]}$ 。失氧反应是锂离子电池热失 控的一个重要原因, 因此, 可以通过抑制氧气释放 来提高电池安全性, 亦即需要改善正极材料的热稳 定性。

脱锂过程伴随着氧空位的产生，氧空位形成后 $\mathrm{Ni}$ 原子将迁移到 $\mathrm{Li}$ 位, 即氧空位加剧了阳离子混排, 反之亦然。理论计算结果表明 ${ }^{[111]}, \mathrm{Al}$ 掺杂可以抑制 氧空位的形成, 即增强了氧稳定性。而 $\mathrm{Mg}$ 可以阻 止 $\mathrm{Ni}$ 的迁移并减少阳离子混排，增强结构稳定性。 同时, 即使已有氧空位存在, $\mathrm{Al}$ 和 $\mathrm{Mg}$ 掺杂也可以阻 止 $\mathrm{Ni}$ 原子的迁移 ${ }^{[112]}$ 。有研究表明, $\mathrm{Al}$ 可以有效地 将电子转移到 $\mathrm{O}$ 中, 而氧电荷的增加使其结合能更 $大^{[113]}$, 即 $\mathrm{Al}-\mathrm{O}$ 键能更大, 掺杂 $\mathrm{Al}$ 之后的 $\mathrm{NCM}$ 结 构更稳定 ${ }^{[114]}$ 。与上述机理不同, Kim 等 ${ }^{[115]}$ 发现, W 掺杂 NCM 的表面会生成一层岩盐相, 通常这种岩 盐相出现在多次循环后或因其他原因被破坏的 $\mathrm{NCM}$ 表面, 在新制备的材料中很少出现。计算结果 表明, 岩盐相中的 $\mathrm{O}$ 结合能比层状相中更高, 即表 面的岩盐相可以减少失氧。此外, 受价态平衡的限 制, 部分 $\mathrm{Ni}^{3+}$ 被还原为 $\mathrm{Ni}^{2+}$, 减少了 Jahn-Teller 活性 点, 对稳定结构有积极作用。掺杂 $1 \mathrm{~mol} \% \mathrm{~W}$ 的 $\mathrm{LNO}$ $\left(\mathrm{LiNiO}_{2}\right) 、 \mathrm{NC} 8911\left(\mathrm{LiNi}_{0.89} \mathrm{Co}_{0.11} \mathrm{O}_{2}\right) 、 \mathrm{NCM} 900505$ 和 NCM801505 的循环性能均得到不同幅度的提升 (图 8(a))。高镍正极材料掺杂 $\mathrm{W}$ 之后热稳定性显著 提升, 最高可超过 $25{ }^{\circ} \mathrm{C}$, 并且这些热反应释放的 总热量也有不同程度的降低(图 8(b))。

使用 $\mathrm{Mo}$ 掺杂时, $\mathrm{Mo}^{6+}$ 倾向于占据 $\mathrm{Mn}^{4+}$ 位, 为 保持电荷平衡, $\mathrm{Ni}^{2+} / \mathrm{Ni}^{3+}$ 的比值会增加, 进而导致锂 镍混排的加剧 ${ }^{[116]}$, 但 Mo 掺杂可以极大地提高材料 的热稳定性。 $\mathrm{Li}_{0.2} \mathrm{Ni}_{0.8} \mathrm{Mn}_{0.1} \mathrm{Co}_{0.1} \mathrm{O}_{2}$ 发生尖晶石相向 岩盐相转变的相转变温度为 $250{ }^{\circ} \mathrm{C}$, 而 $4 \mathrm{~mol} \% \mathrm{Mo}$ 掺杂可将此温度提高到 $350{ }^{\circ} \mathrm{C}$ 以上，同时氧气释放 量大幅减少。原因是 $\mathrm{Mo}^{6+}$ 没有 $\mathrm{d}$ 轨道电子, 根据晶 体场理论, 不存在八面体位置稳定能。因此当温度 升高时, $\mathrm{Mo}^{6+}$ 很容易由八面体位置前移至四面体位 置, 即发生层状向尖晶石结构的相转变。但由于 $\mathrm{Mo}^{6+}$ 在四面体中更稳定, 即含 $\mathrm{Mo}$ 的尖晶石结构更 稳定，因此当温度继续升高时，由尖晶石向岩盐相 的相转变很难发生。Mo 掺杂增大了 NCM622 的晶 胞参数, 可以增强阳离子有序度并拓宽锂离子迁移 通道 ${ }^{[117]}$ 。此外, $\mathrm{Mo}^{6+} / \mathrm{Mo}^{4+}$ 电对能够提升部分放电比 容量, 而较强的 $\mathrm{Mo}-\mathrm{O}$ 键可以抑制晶格氧的流失 

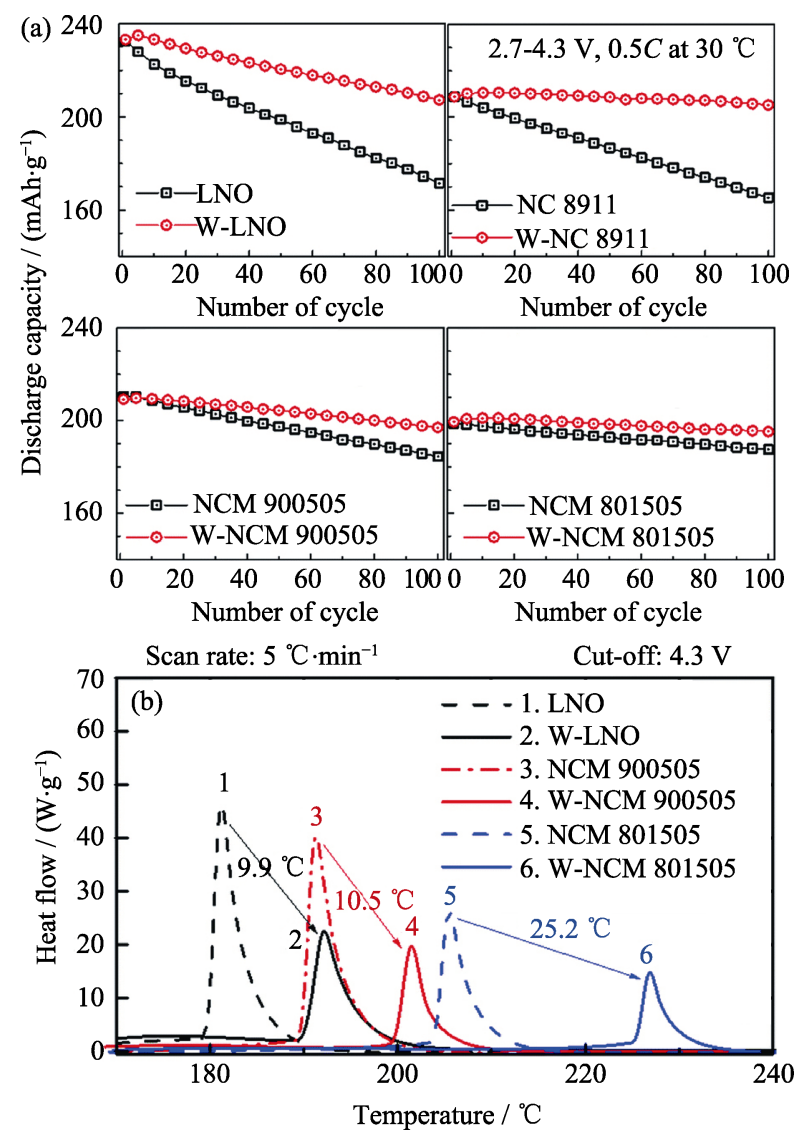

图 8 不同正极材料的(a)循环性能和(b) DSC 曲线 [115]

Fig. 8 (a) Cycling performance and (b) DSC results of different cathodes ${ }^{[115]}$

并稳定材料的表面。因此, Mo 掺杂是提高材料理化 性能的有效方法, 可提高 NCM622 在高压下的电化 学性能。

\section{4 小结}

不同掺杂元素占据的晶格点位有所区别, 而取 代不同点位对 NCM 性能的改善也有所不同。Ta 对 $\mathrm{NCM} 811$ 的高温 $\left(45{ }^{\circ} \mathrm{C}\right)$ 放电比容量和循环性能提 升、电压滞后降低等有益处; $\mathrm{Zr} 、 \mathrm{Mg}$ 掺杂时材料的 $c$ 轴变化率更小, 循环性能更佳, 但倍率较差; 使用 $\mathrm{Al} 、 \mathrm{Ti} 、 \mathrm{Si}$ 进行掺杂时循环性能也有一定程度的提 高，但提升效果不如 $\mathrm{Ta} 、 \mathrm{Zr} 、 \mathrm{Mg}^{[118]}$ 。必要时，可 以采用多元素共掺杂的方式。取代 $\mathrm{Li}$ 位的 $\mathrm{Na}$ 在扩 大锂层层间距的同时, 也有加强结构稳定性的作用, 而掺杂在 $\mathrm{O}$ 位的 $\mathrm{F}$ 则有利于增强晶型和界面稳定性, 同时进行 $\mathrm{Na}$ 和 $\mathrm{F}$ 掺杂时，两者的协同作用能够全面 提升 NCM622 的性能, 如首周库仑效率、倍率、循 环等 ${ }^{[119]}$ 。 $\mathrm{Mg}-\mathrm{Al}-\mathrm{B}$ 共掺杂 ${ }^{[120]}$ 时, $\mathrm{Mg}^{2+}$ 可以增加层 间距，稳定晶体结构; B 起到抑制不稳定相的作用, 同时又不影响 $\mathrm{Li}^{+}$的迁移。 $\mathrm{Mg}-\mathrm{Al}-\mathrm{B}$ 共掺杂能够降 低阳离子混排，提高材料高电压下的界面稳定性。 综上, 在对 NCM 进行掺杂改性, 需要综合考虑掺杂
元素对材料性能的影响，根据不同的需求选择掺杂 元素，必要时需考虑不同掺杂元素的协同作用。

\section{3 掺杂包覆改性}

表面包覆主要是利用惰性物质隔离正极材料表 面与电解液之间的副反应、抑制金属离子的溶解、 优化材料的循环性能，或使用能够与 $\mathrm{Li}_{2} \mathrm{CO}_{3} / \mathrm{LiOH}$ 反应的物质降低残碱、提高材料的安全性能，但基 本不会改变材料的晶体结构, 锂镍混排等缺陷依然 存在。掺杂主要是利用外加元素的离子半径和价态 等性质与过渡金属 $(\mathrm{Ni} 、 \mathrm{Co} 、 \mathrm{Mn})$ 不同，改变材料的 晶格参数和过渡金属价态，提高正极材料的稳定 性、循环性能或倍率性能等, 但对材料表面的改善 有限, 正极材料仍然直接暴露在电解液中。因此, 有 必要将掺杂和包覆结合, 全面提升材料的性能。

\section{1 自发形成的掺杂包覆}

事实证明，在进行掺杂或包覆处理时，两种改 性往往同时起作用 ${ }^{[121]}$ 。例如, $\mathrm{Li}$ 等 ${ }^{[122]}$ 在使用 $\mathrm{Zr}$ 对 NCM811 进行掺杂改性时发现，材料表面会形成一 层 1 2 $\mathrm{nm}$ 的 $\mathrm{Li}_{2} \mathrm{ZrO}_{3}$ 包覆层。 $\mathrm{Zr}$ 的掺杂和包覆双重 作用使 NCM811 具有更优的倍率性能和循环性能 ${ }^{[123]}$ 。 由于低镍材料具有更好的稳定性, 因此可以设计内 部富 $\mathrm{Ni}$ 表面富 $\mathrm{Co} 、 \mathrm{Mn}$ 的梯度材料, 同时提升电性 能和结构性能 ${ }^{[124-126]}$ 。有意思的是，自发形成掺杂/ 包覆双重效果时, 掺杂元素在体相中往往呈梯度分 布，梯度分布的 $\mathrm{Ge}$ 元素能够抑制阳离子混排，同时 还有利于改善 $\mathrm{Li}^{+}$离子传输通道 ${ }^{[127]}$ 。阴离子掺杂时 也有类似效果, Ran 等 ${ }^{[128]}$ 使用 $\mathrm{NH}_{4} \mathrm{H}_{2} \mathrm{PO}_{4}$ 改性 NCM622 时, 得到了 $\mathrm{PO}_{4}{ }^{3-}$ 梯度掺杂和 $\mathrm{Li}_{3} \mathrm{PO}_{4}$ 包覆 的材料, 极大地提升了 NCM622 的高温高电压循环 性能。这是因为 $\mathrm{PO}_{4}{ }^{3}$-掺杂进入氧层稳定了晶体结构, 表面的 $\mathrm{Li}_{3} \mathrm{PO}_{4}$ 包覆层一则可以阻止过渡金属溶解, 二则作为快离子导体改善了离子传输。使用 $\mathrm{Al} 、 \mathrm{Y}$ 、 $\mathrm{Si} 、 \mathrm{Cd} 、 \mathrm{~V}$ 等元素进行改性时, 也有类似效果 ${ }^{[70,129-132]}$ 。 梯度掺杂还能够在正极材料的表面形成一层无序的 层状结构(图 9), 这种结构比岩盐相更有利于锂离子 的传输, 掺杂元素又进一步提高了氧骨架的坚固性, 形成了特殊的掺杂/包覆结构 ${ }^{[133-134]}$ 。

\section{2 控制制备工艺形成掺杂包覆}

除自发形成的掺杂包覆效果外，不同的后处理 工艺可以控制改性方式。通过湿法在 NCM811 表面 沉积一层包覆物, 当焙烧温度 $<700{ }^{\circ} \mathrm{C}$ 时, 形成 $\mathrm{ZrO}_{2}$ 包覆; $>700{ }^{\circ} \mathrm{C}$ 时, 则是 $\mathrm{ZrO}_{2}$ 包覆和 $\mathrm{Zr}$ 掺杂 ${ }^{[135]}$ 。 使用 Ti 对 NCM811 进行改性时, 将表面沉积有无定 


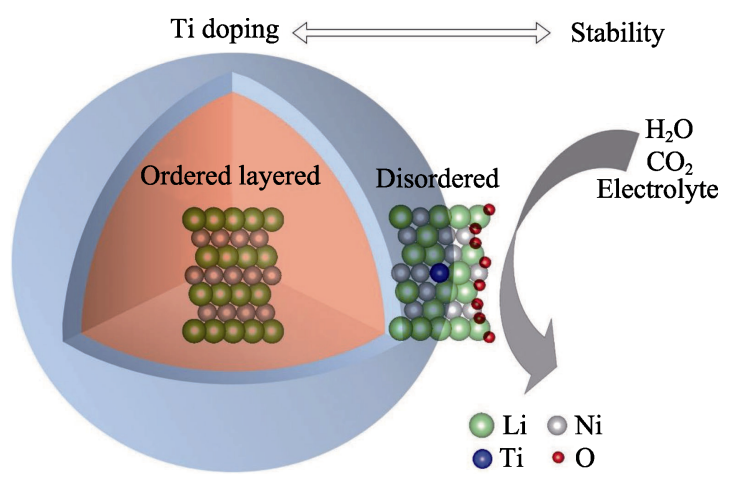

图 9 无序层状相包覆层的示意图 ${ }^{[133]}$

Fig. 9 Schematic illustration of the disordered layered phase coating layer ${ }^{[133]}$

形 $\mathrm{TiO}_{2}$ 的前驱体直接进行配锂焙烧, 得到 $\mathrm{Ti}$ 掺杂 和 $\mathrm{Li}_{2} \mathrm{TiO}_{3}$ 包覆的 $\mathrm{NCM} 811$ 。如将同样的前驱体进 行 $480{ }^{\circ} \mathrm{C}$ 预处理后再配锂焙烧, 则得到 $\mathrm{Li}_{2} \mathrm{TiO}_{3}$ 包 覆的 $\mathrm{NCM} 811^{[136]} 。 \mathrm{Ce}^{4+}$ 很容易将 $\mathrm{Ni}^{2+}$ 氧化为 $\mathrm{Ni}^{3+}$, 可以降低锂镍混排, 并且较强的 $\mathrm{Ce}-\mathrm{O}$ 键对高脱锂 态下的层状结构有很好的稳定作用; 当热处理温度 高于 $500{ }^{\circ} \mathrm{C}$ 时, 表面生成 $\mathrm{CeO}_{2}$ 包覆层, 可以隔离电 解液侵蚀，降低循环过程中表面电阻的增加速度 ${ }^{[137]}$ 。 焙烧气氛对改性效果也有影响 ${ }^{[138]}$ : 在空气气氛下 焙烧时, $\mathrm{Li}_{2} \mathrm{ZrO}_{3}$ 仅存在于 $\mathrm{NCM} 622$ 的表面, 呈岛状 结晶分布; 而当使用氧气气氛时, 得到的是无定型 $\mathrm{Li}_{2} \mathrm{ZrO}_{3}$ 包覆层，且 $\mathrm{Zr}$ 扩散进入体相，可以同时实现 包覆和掺杂的双重效果。虽然高镍材料中含有 $\mathrm{Mn}$ 元素, 但使用 $\mathrm{Mn}$ 化合物对高镍材料进行处理形成 梯度高镍材料, 能够显著提升材料的倍率及循环性 能 ${ }^{[139]}$ 。

掺杂和包覆也可选用不同的元素。聚吡咯(PPy) 是一种典型的导电聚合物, 包覆后能够形成一层导 电网络, 有利于电子/离子扩散, 并可用作正极材料 和电解质之间的保护层。文献[140]报道, $\mathrm{Zr}$ 掺杂可 以改善结构稳定性，提升 NCM523 的常温循环容量 保持率, 再进行 PPy 包覆, 可以提高界面导电性并 抑制界面副反应, 表现出更好的循环性能。研究表 明, $\mathrm{Ti}$ 元素相对于 $\mathrm{La}$ 更容易进入 $\mathrm{NCM}$ 晶格, 因此 将 $\mathrm{Ti}$ 和 $\mathrm{La}$ 与 $\mathrm{NCM} 811$ 一起热处理时, 得到的是 $\mathrm{Ti}$ 掺杂和 $\mathrm{La}_{4} \mathrm{NiLiO}_{8}$ 包覆的材料 ${ }^{[141]}$ 。取代 $\mathrm{Ni}^{2+}$ 位的 $\mathrm{Ti}^{4+}$ 可以降低 $\mathrm{Li} / \mathrm{Ni}$ 混排、抑制循环过程中的相变和 微裂纹的形成, $\mathrm{La}_{4} \mathrm{NiLiO}_{8}$ 除能够抑制循环期间活性 材料表面的结构恶化外, 其优异的电子导电性则有 利于快速电子传输。更重要的是, 由于 $\mathrm{Ti}$ 和 $\mathrm{La}_{4} \mathrm{NiLiO}_{8}$ 降低相变和增强表面稳定性的协同效应, 成功抑制 了 NCM811 内部微裂纹的形成和表面结构恶化。由 于包覆层是通过表面残余添加剂与残锂反应后生成
的，因此大多情况下是不完全包覆，为实现完全包 覆，可再用其他类型的添加剂进行二次包覆 ${ }^{[142]}$ 。

\section{3 小结}

与单独进行包覆或掺杂改性相同, 掺杂/包覆双 改性并不一定能全面提升材料性能。 $\mathrm{Al}_{2} \mathrm{O}_{3}$ 包覆 $/ \mathrm{Mg}^{2+}$ 掺杂的 $\mathrm{NCA}$, 由于 $\mathrm{Al}^{3+}$ 和 $\mathrm{Mg}^{2+}$ 并不具有电化 学活性, 因此其首周放电比容量明显下降, 但这种 改性方式对提升材料结构稳定性十分有效 ${ }^{[143]}$ 。Ge 梯度掺杂和 $\mathrm{Li}_{2} \mathrm{GeO}_{3}$ 包覆 NCM821206 则能同时提 升其循环性能和倍率性能 ${ }^{[127]}$ 。原因是 $\mathrm{Li}_{2} \mathrm{GeO}_{3}$ 包覆 层能够抑制界面副反应以增强正极材料的表面结构 稳定性, Ge 梯度掺杂则不仅可以通过增加相变能垒 抑制层状向尖晶石状或岩盐相的相转变, 而且还可 以通过降低扩散阻力促进 $\mathrm{Li}^{+}$的快速扩散, 减缓电 压衰减。因此, 选择掺杂和包覆元素需十分慎重, 选 择双改性路线时不应只是简单地选择文献报道的掺 杂物和包覆物, 或是简单地单独考虑包覆或掺杂效 果，而是应从掺杂和包覆物的协同作用入手，选择 两种相辅相成的材料, 既实现掺杂和包覆的双重优 点, 又不至于严重降低活性材料的某些性能 ${ }^{[144]}$ 。

\section{4 总结与展望}

随着国家新能源汽车补贴新标准对能量密度要 求的不断攀升, 高镍、特别是镍含量超过 $80 \%$ 的三 元材料被认为是现阶段最有希望的正极材料。但高 镍材料本身的缺点严重制约了其在动力电池中的应 用。各国的研究者们在提高高镍材料的安全性能、 循环性能等方面开展了大量工作, 包覆、掺杂等改 性手段也取得了不同程度的进展。但距离实现镍含 量超过 $80 \%$ 的三元材料在动力电池中大规模应用, 在提升材料性能方面还需进行更深入的探索。

由于正极材料的性能(如粒度分布、元素组成等) 继承自前驱体，因此前驱体的各项技术指标尤为重 要, 但现阶段研究工作对正极材料的设计和合成工 艺优化关注较多，而对前驱体与正极材料性能之间 的关系研究较少, 因此需要结合前驱体的制备技术 和残碱产生的原理设计正极材料及制备工艺，从根 本上解决正极材料的某些问题(如残碱)。

高镍三元正极材料具有高能量密度, 可大规模 应用于新能源汽车领域, 但阳离子混排导致了结构 不稳定和锂离子扩散缓慢, 影响其循环稳定性和倍 率性能。尤其是高度脱锂状态下的结构不稳定性, 更是安全问题的关键所在。除体相结构外，稳定的 界面，如材料表面与电解液的副反应及表面失氧问 
题, 对于提升 $\mathrm{NCM} / \mathrm{NCA}$ 的电化学性能与安全性能 也至关重要。此外, 实际应用还要考虑体积能量密 度, 需进一步解决该问题以增加受限空间中的能量 密度。在过去的研究中, 科学家基于对结构一性能 关系的理解, 通过整合不同的元素降低阳离子混 排、提高结构稳定性, 通过惰性包覆层减少与电解 液的副反应。事实证明, 采用包覆/掺杂改性的手段, 结合对一次粒子形貌的控制(如单晶、特殊结晶取向 等), 能够全方位提高 $\mathrm{NCM} / \mathrm{NCA}$ 在反复充放电循环 中的稳定性, 加快高能量密度正极材料的发展步伐。

目前对改性结果的讨论较多, 而对包覆/掺杂元 素的理论研究不足, 从而导致使用同一种元素进行 改性时, 可能会得到完全不同的效果, 由此带来相 左的机理解释。因此需要研究者系统研究不同价态、 不同半径的元素改性不同镍钴锰比例的三元材料时 的规律, 以及不同热处理工艺对包覆/掺杂效果的影 响规律。更重要的是, 需要将包覆和掺杂两种改性 工艺有机结合, 全面提升高镍正极材料的性能, 而 不是以牺牲其他性能为代价来提升某方面性能。例 如, $\mathrm{Xu}$ 等 ${ }^{[145]}$ 利用氧化化学气相沉积( oCVD)在一次 颗粒和二次颗粒表面同时包覆一层聚乙撑二氧噻吩 (PEDOT), 不仅增强了锂离子和电子传输, 还能够 显著抑制层状到尖晶石/岩盐的相变和失氧、减轻晶 间/晶内裂纹产生, 并有效稳定活性材料一电解质界 面, 全面提升高镍 NCM 高电压下的电化学性能和 热稳定性。改性元素种类选择、改性工艺优化, 包 覆厚度/掺杂深度控制等对于正极材料性能的提升 至关重要, 需要进一步的理解, 并开发新颖的方法 和工艺。

成分梯度有望成为下一步的技术解决方案，梯 度元素的选择和梯度分布有待进一步优化, 以平衡 能量密度和循环稳定性。虽然很容易得到浓度梯度 的 NCM 材料, 并在循环中保持, 但梯度分布的 NCA 却很难制备, 在高温时前驱体的浓度梯度趋于 消失。因此在主体材料的选择和制备工艺研究方面, 还需给予更多的关注。对于梯度掺杂而言, 循环过 程中梯度元素的演变规律, 以及对正极材料性能的 影响, 也需要进行更为深入的研究。

此外, 正极材料的性能最终要体现在全电池中, 相同性质的 $\mathrm{NCM} / \mathrm{NCA}$ 材料匹配不同负极时会面临 不同的失效机制。因此有必要将各种改性的 $\mathrm{NCM} / \mathrm{NCA}$ 材料组装成商品化电池, 并研究其性能 和失效机制, 这有助于为正极材料的改进提供指 导。对于负极材料而言, SEI (Solid Electrolyte Interface 固体电解质界面相)膜的发现和引入极大地 推进了石墨负极的商业化应用。虽然对正极 CEI
(Cathode Electrolyte Interface 正极电解质中间相)膜 的讨论由来已久, 但由于 CEI 膜的形成受众多因素 影响且在高电压下不稳定, 不同研究人员关于 CEI 膜的研究结果之间存在差异。特别是对高镍材料的 研究, 则更为不足。因此, 在 CEI 膜的形成机制和 组成、正极材料本征性质对 CEI 膜的影响规律、高 电压充放电过程中的稳定性与变化规律、以及 CEI 与 SEI 膜之间的相互影响机制等方面, 需要开展更 为深入和广泛的研究。

最后, 更重要的是在以上研究基础之上, 开发 适合工业生产的工艺, 再配合电池技术的进步, 实 现动力电池性能的全面提升。相信在学术界和工业 界的合作努力下, 高安全性能、高能量密度的动力 电池将会推动新能源汽车的持续发展。

\section{参考文献:}

[1] CHOI N S, CHEN Z, FREUNBERGER S A, et al. Challenges facing lithium batteries and electrical double-layer capacitors. Angew. Chem. Int. Ed., 2012, 51(40): 9994-10024.

[2] LIU W, OH P, LIU X, et al. Nickel-rich layered lithium transitionmetal oxide for high energy lithium-ion batteries. Angew. Chem. Int. Ed., 2015, 54(15): 4440-4457.

[3] SCROSATI B, GARCHE J. Lithium batteries: status, prospects and future. J. Power Sources, 2010, 195(9): 2419-2430.

[4] 柏祥涛, 孙学义, 庄卫东, 等. 氢氧化物前驱体制备 $\mathrm{LiNi}_{0.5} \mathrm{Co}_{0.2} \mathrm{Mn}_{0.3} \mathrm{O}_{2}$ 的机理. 电池, 2014, 44(5): 260-263.

[5] LEE K S, MYUNG S T, AMINE K, et al. Structural and electrochemical properties of layered $\mathrm{Li}\left[\mathrm{Ni}_{1-2 x} \mathrm{Co}_{x} \mathrm{Mn}_{x}\right] \mathrm{O}_{2} \quad(x=0.1-0.3)$ positive electrode materials for Li-ion batteries. J. Electrochem. Soc., 2007, 154(10): A971-A977.

[6] JOUANNEAU S, MACNEIL D D, LU Z, et al. Morphology and safety of $\mathrm{Li}\left[\mathrm{Ni}_{x} \mathrm{Co}_{1-2 x} \mathrm{Mn}_{x}\right] \mathrm{O}_{2}(0 \leqslant x \leqslant 1 / 2)$. J. Electrochem. Soc., 2003, 150(10): A1299-1304.

[7] MACNEIL D D, LU Z H, CHEN Z H, et al. A comparison of the electrode/electrolyte reaction at elevated temperatures for various Li-ion battery cathodes. J. Power Sources, 2002, 108(1/2): 8-14.

[8] KIM J, LEE H, CHA H, et al. Nickel-rich cathodes: prospect and reality of Ni-rich cathode for commercialization. Adv. Energy Mater., 2018, 8(6): 1702028.

[9] XIONG X H, WANG Z X, YUE P, et al. Washing effects on electrochemical performance and storage characteristics of $\mathrm{LiNi}_{0.8} \mathrm{Co}_{0.1} \mathrm{Mn}_{0.1} \mathrm{O}_{2}$ as cathode material for lithium-ion batteries. $J$. Power Sources, 2013, 222: 318-325.

[10] XU S, WANG X, ZHANG W, et al. The effects of washing on $\mathrm{LiNi}_{0.83} \mathrm{Co}_{0.13} \mathrm{Mn}_{0.04} \mathrm{O}_{2}$ cathode materials. J. Solid State Ionics, 2019, 334: 105-110.

[11] LI J, CHEN B R, ZHOU H M. Effects of washing and heat-treatment on structure and electrochemical charge/discharge property of $\mathrm{LiNi}_{0.8} \mathrm{Co}_{0.15} \mathrm{Al}_{0.05} \mathrm{O}_{2}$ powder. J. Inorg. Mater., 2016, 31(7): 773-778.

[12] XU S Z, LUO G F, JACOBS R, et al. Ab initio modeling of electrolyte molecule ethylene carbonate decomposition reaction on $\mathrm{Li}(\mathrm{Ni}, \mathrm{Mn}, \mathrm{Co}) \mathrm{O}_{2}$ cathode surface. ACS Appl. Mater. Interfaces, 2017, 9: 20545-20553.

[13] MYUNG S T, IZUMI K, KOMABA S, et al. Role of alumina coating on $\mathrm{Li}-\mathrm{Ni}-\mathrm{Co}-\mathrm{Mn}-\mathrm{O}$ particles as positive electrode material 
for lithium-ion batteries. Chem. Mater., 2005, 17(14): 3695-3704.

[14] YU H J, QIAN Y M, OTANI M, et al. Study of the lithium/nickel ions exchange in the layered $\mathrm{LiNi}_{0.42} \mathrm{Mn}_{0.42} \mathrm{Co}_{0.16} \mathrm{O}_{2}$ cathode material for lithium ion batteries: experimental and first-principles calculations. Energy Environ. Sci., 2014, 7: 1068-1078.

[15] AURBACH D. Electrode-solution interactions in Li-ion batteries: a short summary and new insights. J. Power Sources, 2003, 119-121: 497-503.

[16] MYUNG S T, AMINE K, SUN Y K. Surface modification of cathode materials from nano- to microscale for rechargeable lithiumion batteries. J. Mater. Chem., 2010, 20: 7074-7095.

[17] YOON W S, HANSON J, MCBREEN J, et al. A study on the newly observed intermediate structures during the thermal decomposition of nickel-based layered cathode materials using time-resolved XRD. Electrochem.Commun., 2006, 8: 859-862.

[18] KONISHI H, YUASA T, YOSHIKAWA M. Thermal stability of $\mathrm{Li}_{1-y} \mathrm{Ni}_{x} \mathrm{Mn}_{(1-x) / 2} \mathrm{Co}_{(1-x) / 2} \mathrm{O}_{2}$ layer-structured cathode materials used in Li-ion batteries. J. Power Sources, 2011, 196: 6884-6888.

[19] BAK S M, HU E Y, ZHOU Y N, et al. Structural changes and thermal stability of charged $\mathrm{LiNi}_{x} \mathrm{Mn}_{y} \mathrm{Co}_{z} \mathrm{O}_{2}$ cathode materials studied by combined in situ time-resolved XRD and mass spectroscopy. ACS Appl. Mater. Interfaces, 2014, 6(24): 22594-22601.

[20] JUNG S K, GWON H, HONG J, et al. Understanding the degradation mechanisms of $\mathrm{LiNi}_{0.5} \mathrm{Co}_{0.2} \mathrm{Mn}_{0.3} \mathrm{O}_{2}$ cathode material in lithium ion batteries. Adv. Energy Mater., 2014, 4: 1300787.

[21] 邵奕嘉, 黄斌, 刘全兵, 等. 三元镍钴锰正极材料的制备及改 性. 化学进展, 2018, 30(4): 410-419.

[22] MANTHIRAM A, KNIGHT J C, MYUNG S T, et al. Nickel-rich and lithium-rich layered oxide cathodes: progress and perspectives. Adv. Energy Mater., 2016, 6(1): 1501010.

[23] CHEN Z, CHAO D, LIN J, et al. Recent progress in surface coating of layered $\mathrm{LiNi}_{x} \mathrm{Co}_{y} \mathrm{Mn}_{z} \mathrm{O}_{2}$ for lithium-ion batteries. Mater. Res. Bull., 2017, 96(4): 491-502.

[24] AURBACH D. The electrochemical behavior of lithium salt solutions of $\gamma$-butyrolactone with noble metal electrodes J. Electrochem. Soc., 1989, 136(4): 906-913.

[25] EDSTROM K, GUSTAFSSON T, THOMAS J. The cathodeelectrolyte interface in the Li-ion battery. Electrochim. Acta, 2004, 50(2/3): 397-403.

[26] AURBACH D. Identification of surface films formed on lithium surfaces in $\gamma$-butyrolactone solutions II. contaminated solutions. $J$. Electrochem. Soc., 1989, 136(6): 1611-1614.

[27] LIN F, MARKUS I M, NORDLUND D, et al. Surface reconstruction and chemical evolution of stoichiometric layered cathode materials for lithium-ion batteries. Nat. Commun., 2014, 5: 3529.

[28] LI J, LIU H, XIA J, et al. The impact of electrolyte additives and upper cut-off voltage on the formation of a rocksalt surface layer in $\mathrm{LiNi}_{0.8} \mathrm{Mn}_{0.1} \mathrm{Co}_{0.1} \mathrm{O}_{2}$ electrodes. J. Electrochem. Soc., 2017, 164(4): A655-A665.

[29] SHI Y, ZHANG M H, QIAN D N, et al. Ultrathin $\mathrm{Al}_{2} \mathrm{O}_{3}$ coatings for improved cycling performance and thermal stability of $\mathrm{LiNi}_{0.5} \mathrm{Co}_{0.2} \mathrm{Mn}_{0.3} \mathrm{O}_{2}$ cathode material. Electrochim. Acta, 2016, 203(10): 154-161.

[30] NEUDECK S, STRAUSS F, GARCIA G, et al. Room temperature, liquid-phase $\mathrm{Al}_{2} \mathrm{O}_{3}$ surface coating approach for Ni-rich layered oxide cathode material. Chem. Commun., 2019, 55: 2174-2177.

[31] VETTER J, NOVAK P, WAGNER M R, et al. Ageing mechanisms in lithium-ion batteries. J. Power Sources, 2005, 147(1/2): 269-281.

[32] AURBACH D, MARKOVSKY B, SALITRA G, et al. Review on electrode-electrolyte solution interactions, related to cathode materials for Li-ion batteries. J. Power Sources, 2007, 165(2): 491-499.
[33] LIANG L W, HU G R, JIANG F, et al. Electrochemical behaviours of $\mathrm{SiO}_{2}$-coated $\mathrm{LiNi}_{0.8} \mathrm{Co}_{0.1} \mathrm{Mn}_{0.1} \mathrm{O}_{2}$ cathode materials by a novel modification method. J. Alloys Compd., 2016, 657: 570-581.

[34] GAN Z G, HU G R, PENG Z D, et al. Surface modification of $\mathrm{LiNi}_{0.8} \mathrm{Co}_{0.1} \mathrm{Mn}_{0.1} \mathrm{O}_{2}$ by $\mathrm{WO}_{3}$ as a cathode material for LIB. Appl. Surf. Sci., 2019, 481: 1228-1238.

[35] LI Y P, YAN G J, LUO L M, et al. Enhanced electrochemical performance of $\mathrm{LiNi}_{0.4} \mathrm{Co}_{0.2} \mathrm{Mn}_{0.4} \mathrm{O}_{2}$ cathode materials via $\mathrm{Y}_{2} \mathrm{O}_{3}$ coating. Mater. Res. Express, 2019, 6(10): 105533.

[36] LOGHAVI M M, MOHAMMADI-MANESH H, EQRA R. $\mathrm{LiNi}_{0.8} \mathrm{Co}_{0.15} \mathrm{Al}_{0.05} \mathrm{O}_{2}$ coated by chromium oxide as a cathode material for lithium-ion batteries. J Solid State Electrochem., 2019, 23(8): 2569-2578.

[37] MYUNG S T, IZUMI K, KOMABA S, et al. Functionality of oxide coating for $\mathrm{Li}\left[\mathrm{Li}_{0.05} \mathrm{Ni}_{0.4} \mathrm{Co}_{0.15} \mathrm{Mn}_{0.4}\right] \mathrm{O}_{2}$ as positive electrode materials for lithium-ion secondary batteries. J. Phys. Chem. C, 2007, 111(10): 4061-4067.

[38] ZUO D X, WANG C P, TIAN G L, et al. Comparative study of $\mathrm{Al}_{2} \mathrm{O}_{3}, \mathrm{SiO}_{2}$ and $\mathrm{TiO}_{2}$-coated $\mathrm{LiNi}_{0.6} \mathrm{Co}_{0.2} \mathrm{Mn}_{0.2} \mathrm{O}_{2}$ electrode prepared by hydrolysis coating technology. J. Electrochem. Sci. Eng., 2019, 9(2): 85-97.

[39] ZHU W C, HUANG X, LIU T T, et al. Ultrathin $\mathrm{Al}_{2} \mathrm{O}_{3}$ coating on $\mathrm{LiNi}_{0.8} \mathrm{Co}_{0.1} \mathrm{Mn}_{0.1} \mathrm{O}_{2}$ cathode material for enhanced cycleability at extended voltage ranges. Coatings, 2019, 9(2): 92.

[40] NEUDECK S, MAZILKIN A, REITZ C, et al. Effect of low-temperature $\mathrm{Al}_{2} \mathrm{O}_{3} \mathrm{ALD}$ coating on Ni-rich layered oxide composite cathode on the long-term cycling performance of lithiumion batteries. Sci. Rep., 2019, 9: 5328.

[41] CUI X L, AI L, MAO L P, et al. Enhanced electrochemical properties of $\mathrm{LiNi}_{0.6} \mathrm{Co}_{0.2} \mathrm{Mn}_{0.2} \mathrm{O}_{2}$ cathode material by the diffusional $\mathrm{Al}_{2} \mathrm{O}_{3}$ coating layer. Ionics, 2019, 25(2): 411-419.

[42] YANG K, FAN L Z, GUO J, et al. Significant improvement of electrochemical properties of $\mathrm{AlF}_{3}$-coated $\mathrm{LiNi}_{0.5} \mathrm{Co}_{0.2} \mathrm{Mn}_{0.3} \mathrm{O}_{2}$ cathode materials. Electrochim. Acta, 2012, 63: 363-368.

[43] LEE S H, YOON C S, AMINE K, et al. Improvement of long-term cycling performance of $\mathrm{Li}\left[\mathrm{Ni}_{0.8} \mathrm{Co}_{0.15} \mathrm{Al}_{0.05}\right] \mathrm{O}_{2}$ by $\mathrm{AlF}_{3}$ coating. J. Power Sources, 2013, 234: 201-207.

[44] CHO Y, OH P, CHO J. A new type of protective surface layer for high-capacity Ni-based cathode materials: nanoscaled surface pillaring layer. Nano Lett., 2013, 13(3): 1145-1152.

[45] HAN B L, LU X C. Effect of nano-sized $\mathrm{CeF}_{3}$ on microstructure, mechanical, high temperature friction and corrosion behavior of Ni-W composite coatings. Surf. Coat. Technol., 2009, 203(23): 3656-3660.

[46] KUMAR D A, SELVASEKARAPANDIAN S, NITHYA H, et al. Structural and conductivity analysis on cerium fluoride nanoparticles prepared by sonication assisted method. Solid State Sci., 2012, 14(5): 626-634.

[47] KUMAR D A, SELVASEKARAPANDIAN S, NITHYA H, et al. Influence of substrate temperature on $\mathrm{CeF}_{3}$ thin films prepared by thermal evaporation. Mater. Chem. Phys., 2014, 143(2): 765-772.

[48] XIE Y, GAO D, ZHANG $\mathrm{L}$ L, et al. $\mathrm{CeF}_{3}$-modified $\mathrm{LiNi}_{1 / 3} \mathrm{Co}_{1 / 3} \mathrm{Mn}_{1 / 3} \mathrm{O}_{2}$ cathode material for high-voltage Li-ion batteries. Ceram. Int., 2016, 42(13): 14587-14594.

[49] SONG H G, KIM S B, PARK Y J. Enhanced electrochemical properties of $\mathrm{Li}_{[}\left[\mathrm{Ni}_{0.5} \mathrm{Co}_{0.2} \mathrm{Mn}_{0.3}\right] \mathrm{O}_{2}$ cathode by surface coating using $\mathrm{LaF}_{3}$ and $\mathrm{MgF}_{2}$. J. Electroceram., 2012, 29(2): 163-169.

[50] DAI S C, YAN G J, WANG L, et al. Enhanced electrochemical performance and thermal properties of Ni-rich $\mathrm{LiNi}_{0.8} \mathrm{Co}_{0.1} \mathrm{Mn}_{0.1} \mathrm{O}_{2}$ cathode material via $\mathrm{CaF}_{2}$ coating. J. Electroanal. Chem., 2019, 847: 113197.

[51] LIM Y J, LEE S M, LIM H, et al. Amorphous Li-Zr-O layer coat- 
ing on the surface of high-Ni cathode materials for lithium ion batteries. Electrochim. Acta, 2018, 282: 311-316.

[52] LIU S J, WU H, HUANG L, et al. Synthesis of $\mathrm{Li}_{2} \mathrm{Si}_{2} \mathrm{O}_{5}$-coated $\mathrm{LiNi}_{0.6} \mathrm{Co}_{0.2} \mathrm{Mn}_{0.2} \mathrm{O}_{2}$ cathode materials with enhanced high-voltage electrochemical properties for lithium-ion batteries. J. Alloys Compd., 2016, 674: 447-454.

[53] JO C H, CHO D H, NOH H J, et al. An effective method to reduce residual lithium compounds on Ni-rich $\mathrm{Li}\left[\mathrm{Ni}_{0.6} \mathrm{Co}_{0.2} \mathrm{Mn}_{0.2}\right] \mathrm{O}_{2}$ active material using a phosphoric acid derived $\mathrm{Li}_{3} \mathrm{PO}_{4}$ nanolayer. Nano Res., 2015, 8(5): 1464-1479.

[54] ZHU J, LI Y J, XUE L L, et al. Enhanced electrochemical performance of $\mathrm{Li}_{3} \mathrm{PO}_{4}$ modified $\mathrm{Li}\left[\mathrm{Ni}_{0.8} \mathrm{Co}_{0.1} \mathrm{Mn}_{0.1}\right] \mathrm{O}_{2}$ cathode material via lithium-reactive coating. J. Alloys Compd., 2019, 773: 112-120.

[55] FAN Q L, YANG S D, LIU J, et al. Mixed-conducting interlayer boosting the electrochemical performance of Ni-rich layered oxide cathode materials for lithium ion batteries. J. Power Sources, 2019, 421: 91-99.

[56] BAN L Q, YIN Y P, ZHUANG W D, et al. Electrochemical performance improvement of $\mathrm{Li}_{1.2}\left[\mathrm{Mn}_{0.54} \mathrm{Ni}_{0.13} \mathrm{Co}_{0.13}\right] \mathrm{O}_{2}$ cathode material by sulfur incorporation. Electrochim. Acta, 2015, 180: 218-226.

[57] HUANG Y Q, HUANG Y H, HU X L. Enhanced electrochemical performance of $\mathrm{LiNi}_{0.8} \mathrm{Co}_{0.15} \mathrm{Al}_{0.05} \mathrm{O}_{2}$ by nanoscale surface modification with $\mathrm{Co}_{3} \mathrm{O}_{4}$. Electrochim. Acta, 2017, 231: 294-299.

[58] XU S, DU C Y, XU X, et al. A mild surface washing method using protonated polyaniline for Ni-rich $\mathrm{LiNi}_{0.8} \mathrm{Co}_{0.1} \mathrm{Mn}_{0.1} \mathrm{O}_{2}$ material of lithium ion batteries. Electrochim. Acta, 2017, 248: 534-540.

[59] HE X S, HAN G K, LOU S F, et al. Improved electrochemical performance of $\mathrm{LiNi}_{0.8} \mathrm{Co}_{0.15} \mathrm{Al}_{0.05} \mathrm{O}_{2}$ cathode material by coating of graphene nanodots. J. Electrochem. Soc., 2019, 166(6): A1038-A1044.

[60] YANG H, WU K P, HU G R, et al. Design and synthesis of doublefunctional polymer composite layer coating to enhance the electrochemical performance of the Ni-rich cathode at the upper cutoff voltage. ACS Appl. Mater. Interfaces, 2019, 11: 8556-8566.

[61] GAN Q M, QIN N, ZHU Y H, et al. Polyvinylpyrrolidone-induced uniform surface-conductive polymer coating endows Ni-rich $\mathrm{LiNi}_{0.8} \mathrm{Co}_{0.1} \mathrm{Mn}_{0.1} \mathrm{O}_{2}$ with enhanced cyclability for lithium-ion batteries. ACS Appl. Mater. Interfaces, 2019, 11(13): 12594-12604.

[62] 尹艳萍, 庄卫东, 王忠, 等. 一种锂离子电池用含镍层状正极材 料/碳复合材料及其制备方法. 中国, CN108232153A. 2018.06.29.

[63] 王忠, 庄卫东, 尹艳萍, 等. 一种石墨烯改性锂镍钴锰氧化物 正极材料及其制备方法. 中国, CN109244448A. 2019.01.18.

[64] 王忠, 庄卫东, 尹艳萍, 等. 一种纳米碳材料改性锂镍钴锰氧 化物正极材料及制备方法. 中国, CN109473642A. 2019.03.15.

[65] ZHANG S S, CHEN J, WANG C S. Elemental sulfur as a cathode additive for enhanced rate capability of layered lithium transition metal oxides. J. Electrochem. Soc., 2019, 166(4): A487-A492.

[66] ZHANG S S, FAN X L, WANG C S. Enhanced electrochemical performance of Ni-rich layered cathode materials by using $\mathrm{LiPF}_{6}$ as a cathode additive. ChemElectroChem, 2019, 6(5): 1536-1541.

[67] LI J, DOWNIE L E, MA L, et al. Study of the failure mechanisms of $\mathrm{LiNi}_{0.8} \mathrm{Mn}_{0.1} \mathrm{Co}_{0.1} \mathrm{O}_{2}$ cathode material for lithium ion batteries. $J$ Electrochem. Soc., 2015, 162(7): A1401-A1408.

[68] LIU H, WOLF M, KARKI K, et al. Intergranular cracking as a major cause of long-term capacity fading of layered cathodes. Nano Lett., 2017, 17(6): 3452-3457.

[69] ZHAO E Y, CHEN M M, HU Z B, et al. Improved cycle stability of high-capacity Ni-rich $\mathrm{LiNi}_{0.8} \mathrm{Mn}_{0.1} \mathrm{Co}_{0.1} \mathrm{O}_{2}$ at high cut-off voltage by $\mathrm{Li}_{2} \mathrm{SiO}_{3}$ coating. J. Power Sources, 2017, 343: 345-353.

[70] PENG Z J, YANG G W, LI F Q, et al. Improving the cathode properties of Ni-rich $\mathrm{LiNi}_{0.6} \mathrm{Co}_{0.2} \mathrm{Mn}_{0.2} \mathrm{O}_{2}$ at high voltages under $5 C$ by $\mathrm{Li}_{2} \mathrm{SiO}_{3}$ coating and $\mathrm{Si}^{4+}$ doping. J. Alloys Compd., 2018, 762: $827-834$

[71] LEE S W, KIM M S, JEONG J H, et al. $\mathrm{Li}_{3} \mathrm{PO}_{4}$ surface coating on Ni-rich $\mathrm{LiNi}_{0.6} \mathrm{Co}_{0.2} \mathrm{Mn}_{0.2} \mathrm{O}_{2}$ by a citric acid assisted Sol-Gel method: improved thermal stability and high-voltage performance. J. Power Sources, 2017, 360: 206-214.

[72] KIM S B, LEE K J, CHOI W J, et al. Preparation and cycle performance at high temperature for $\mathrm{Li}\left[\mathrm{Ni}_{0.5} \mathrm{Co}_{0.2} \mathrm{Mn}_{0.3}\right] \mathrm{O}_{2}$ coated with $\mathrm{LiFePO}_{4}$. J. Solid State Electrochem., 2010, 14: 919-922.

[73] WU Z Z, JI S P, LIU T C, et al. Aligned $\mathrm{Li}^{+}$tunnels in core-shell $\mathrm{Li}\left(\mathrm{Ni}_{x} \mathrm{Mn}_{y} \mathrm{Co}_{z}\right) \mathrm{O}_{2} @ \mathrm{LiFePO}_{4}$ enhances its high voltage cycling stability as Li-ion battery cathode. Nano Lett., 2016, 16(10): 6357-6363.

[74] ZHU L, YAN T F, JIA D, et al. $\mathrm{LiFePO}_{4}$-coated $\mathrm{LiNi}_{0.5} \mathrm{Co}_{0.2} \mathrm{Mn}_{0.3} \mathrm{O}_{2}$ cathode materials with improved high voltage electrochemical performance and enhanced safety for lithium ion pouch cells. $J$. Electrochem. Soc., 2019, 166(3): A5437-A5444.

[75] KIM W S, KIM S B, JANG I C, et al. Remarkable improvement in cell safety for $\mathrm{Li}\left[\mathrm{Ni}_{0.5} \mathrm{Co}_{0.2} \mathrm{Mn}_{0.3}\right] \mathrm{O}_{2}$ coated with $\mathrm{LiFePO}_{4} . J$. Alloys Compd., 2010, 492(1/2): L87-L90.

[76] DIAO R J, NAYAKA G P, ZHU C Y, et al. $\mathrm{CePO}_{4}$ coated $\mathrm{LiNi}_{0.6} \mathrm{Co}_{0.2} \mathrm{Mn}_{0.2} \mathrm{O}_{2}$ as cathode material and its electrochemical performance. Int. J. Electrochem. Sci., 2019, 14: 8070-8079.

[77] TONG H, DONG P Y, ZHANG J F, et al. Cathode material $\mathrm{LiNi}_{0.8} \mathrm{Co}_{0.1} \mathrm{Mn}_{0.1} \mathrm{O}_{2} / \mathrm{LaPO}_{4}$ with high electrochemical performance for lithium-ion batteries. J. Alloys Compd., 2018, 764: 44-50.

[78] LIU W M, HU G R, DU K, et al. Surface coating of $\mathrm{LiNi}_{0.8} \mathrm{Co}_{0.15} \mathrm{Al}_{0.05} \mathrm{O}_{2}$ with $\mathrm{LiCoO}_{2}$ by a molten salt method. Surf. Coat. Tech., 2013, 216: 267-272.

[79] LIU W M, HU G R, DU K, et al. Synthesis and characterization of $\mathrm{LiCoO}_{2}$-coated $\mathrm{LiNi}_{0.8} \mathrm{Co}_{0.15} \mathrm{Al}_{0.05} \mathrm{O}_{2}$ cathode materials. Mater. Lett., 2012, 83: 11-13.

[80] LIU W M, HU G R, DU K, et al. Enhanced storage property of $\mathrm{LiNi}_{0.8} \mathrm{Co}_{0.15} \mathrm{Al}_{0.05} \mathrm{O}_{2}$ coated with $\mathrm{LiCoO}_{2}$. J. Power Sources, 2013, 230: 201-206.

[81] YAN P F, ZHENG J M, GU M, et al. Intragranular cracking as a critical barrier for high-voltage usage of layer-structured cathode for lithium-ion batteries. Nat. Commun., 2017, 8: 14101.

[82] HASHIGAMI S, KATO Y, YOSHIMI K, et al. Influence of lithium silicate coating on retarding crack formation in $\mathrm{LiNi}_{0.5} \mathrm{Co}_{0.2} \mathrm{Mn}_{0.3} \mathrm{O}_{2}$ cathode particles. Electrochim. Acta, 2018, 291: 304-310.

[83] DU M L, YANG P, HE W X, et al. Enhanced high-voltage cycling stability of Ni-rich $\mathrm{LiNi}_{0.8} \mathrm{Co}_{0.1} \mathrm{Mn}_{0.1} \mathrm{O}_{2}$ cathode coated with $\mathrm{Li}_{2} \mathrm{O}-2 \mathrm{~B}_{2} \mathrm{O}_{3}$. J. Alloys Compd., 2019, 805: 991-998.

[84] HASHIGAMI S, YOSHIMI K, KATO Y, et al. Improvement of cycleability and rate-capability of $\mathrm{LiNi}_{0.5} \mathrm{Co}_{0.2} \mathrm{Mn}_{0.3} \mathrm{O}_{2}$ cathode materials coated with lithium boron oxide by an antisolvent precipitation method. ChemistrySelect, 2019, 4(29): 8676-8681.

[85] ZHANG J Y, CAO Y, OU X, et al. Constituting the NASICON type solid electrolyte coated material forming anti-high voltage system to enhance the high cut-off voltage performance of $\mathrm{LiNi}_{0.6} \mathrm{Co}_{0.2} \mathrm{Mn}_{0.2} \mathrm{O}_{2}$ via charge attracts electrostatic assembly. $J$. Power Sources, 2019, 436: 226722.

[86] PARK K, PARK J H, HONG S G, et al. Enhancement in the electrochemical performance of zirconium/phosphate bi-functional coatings on $\mathrm{LiNi}_{0.8} \mathrm{Co}_{0.15} \mathrm{Mn}_{0.05} \mathrm{O}_{2}$ by the removal of $\mathrm{Li}$ residuals. Phys. Chem. Chem. Phys., 2016, 18: 29076-29085.

[87] HE X S, XU X, WANG L G, et al. Enhanced electrochemical performance of $\mathrm{LiNi}_{0.8} \mathrm{Co}_{0.15} \mathrm{Al}_{0.05} \mathrm{O}_{2}$ cathode material via $\mathrm{Li}_{2} \mathrm{TiO}_{3}$ 
nanoparticles coating. J. Electrochem. Soc., 2019, 166(2): A143-A150.

[88] YANG J, YU Z Y, YANG B, et al. Electrochemical characterization of $\mathrm{Cr}_{8} \mathrm{O}_{21}$ modified $\mathrm{LiNi}_{0.5} \mathrm{Co}_{0.2} \mathrm{Mn}_{0.3} \mathrm{O}_{2}$ cathode material. Electrochim. Acta, 2018, 266: 342-347.

[89] GAO T P, WONG K W, FUNG K Y, et al. A rational three-step calcination strategy for synthesizing high-quality $\mathrm{LiNi}_{0.5} \mathrm{Mn}_{0.3} \mathrm{Co}_{0.2} \mathrm{O}_{2}$ cathode materials: the key role of suppressing $\mathrm{Li}_{2} \mathrm{O}$ formation. Electrochim. Acta, 2018, 288: 153-164.

[90] BHUVANESWARI D, BABU G, KALAISELVI N. Effect of surface modifiers in improving the electrochemical behavior of $\mathrm{LiNi}_{0.4} \mathrm{Mn}_{0.4} \mathrm{Co}_{0.2} \mathrm{O}_{2}$ cathode. Electrochim. Acta, 2013, 109: 684-693.

[91] WANG D, LI X H, WANG Z X, et al. Role of zirconium dopant on the structure and high voltage electrochemical performances of $\mathrm{LiNi}_{0.5} \mathrm{Co}_{0.2} \mathrm{Mn}_{0.3} \mathrm{O}_{2}$ cathode materials for lithium ion batteries. Electrochim. Acta, 2016, 188: 48-56.

[92] POUILLERIE C, CROGUENNEC L, BIENSAN P, et al. Synthesis and characterization of new $\mathrm{LiNi}_{1-y} \mathrm{Mg}_{y} \mathrm{O}_{2}$ positive electrode materials for lithium-ion batteries. J. Electrochem. Soc., 2000, 147(6): 2061-2069.

[93] XIE Q, LI W D, MANTHIRAM A. A Mg-doped high-nickel layered oxide cathode enabling safer, high-energy-density Li-ion batteries. Chem. Mater., 2019, 31(3): 938-946.

[94] POUILlERIE C, CROGUENNEC L, DELMAS C. The $\mathrm{Li}_{x} \mathrm{Ni}_{1-y} \mathrm{Mg}_{y} \mathrm{O}_{2} \quad(y=0.05,0.10)$ system: structural modifications observed upon cycling. Solid State Ionics, 2000, 132(1/2): 15-29.

[95] HUANG B, LIN X H, WANG Z X, et al. Synthesis of Mg-doped $\mathrm{LiNi}_{0.8} \mathrm{Co}_{0.15} \mathrm{Al}_{0.05} \mathrm{O}_{2}$ oxide and its electrochemical behavior in high-voltage lithium-ion batteries. Ceram. Int., 2014, 40(8): 13223-13230.

[96] BREUER O, CHAKRABORTY A, LIU J, et al. Understanding the role of minor molybdenum doping in $\mathrm{LiNi}_{0.5} \mathrm{Co}_{0.2} \mathrm{Mn}_{0.3} \mathrm{O}_{2}$ electrodes: from structural and surface analyses and theoretical modeling to practical electrochemical cells. ACS Appl. Mater. Interfaces, 2018, 10(35): 29608-29621.

[97] WOO S U, PARK B C, YOON C S, et al. Improvement of electrochemical performances of $\mathrm{Li}\left[\mathrm{Ni}_{0.8} \mathrm{Co}_{0.1} \mathrm{Mn}_{0.1}\right] \mathrm{O}_{2}$ cathode materials by fluorine substitution. J. Electrochem. Soc., 2007, 154(7): A649-A655.

[98] LI C L, KAN W H, XIE H L, et al. Inducing favorable cation antisite by doping halogen in Ni-rich layered cathode with ultrahigh stability. Adv. Sci., 2019, 6(4): 1801406.

[99] LI X, XIE Z W, LIU W J, et al. Effects of fluorine doping on structure, surface chemistry, and electrochemical performance of $\mathrm{LiNi}_{0.8} \mathrm{Co}_{0.15} \mathrm{Al}_{0.05} \mathrm{O}_{2}$. Electrochim. Acta, 2015, 174: 1122-1130.

[100] LÜ C J, YANG J, PENG Y, et al. 1D Nb-doped $\mathrm{LiNi}_{1 / 3} \mathrm{Co}_{1 / 3} \mathrm{Mn}_{1 / 3} \mathrm{O}_{2}$ nanostructures as excellent cathodes for Li-ion battery. Electrochim. Acta, 2019, 297: 258-266.

[101] YANG Z G, XIANG W, WU Z G, et al. Effect of niobium doping on the structure and electrochemical performance of $\mathrm{LiNi}_{0.5} \mathrm{Co}_{0.2} \mathrm{Mn}_{0.3} \mathrm{O}_{2}$ cathode materials for lithium ion batteries. Ceram. Int., 2017, 43(4): 3866-3872.

[102] WU J F, LIU H G, YE X H, et al. Effect of Nb doping on electrochemical properties of $\mathrm{LiNi}_{1 / 3} \mathrm{Co}_{1 / 3} \mathrm{Mn}_{1 / 3} \mathrm{O}_{2}$ at high cutoff voltage for lithium-ion battery. J. Alloys Compd., 2015, 644: 223-227.

[103] BREGER J, MENG Y S, HINUMA Y, et al. Effect of high voltage on the structure and electrochemistry of $\mathrm{LiNi}_{0.5} \mathrm{Mn}_{0.5} \mathrm{O}_{2}$ : a joint experimental and theoretical study. Chem. Mater., 2006, 18(20): 4768-4781.

[104] SCHIPPER F, DIXIT M, KOVACHEVA D, et al. Stabilizing nickel-rich layered cathode materials by a high-charge cation doping strategy: zirconium-doped $\mathrm{LiNi}_{0.6} \mathrm{Co}_{0.2} \mathrm{Mn}_{0.2} \mathrm{O}_{2}$. J. Mater.
Chem. A, 2016, 4: 16073-16084.

[105] DONG S D, ZHOU Y, HAI C X, et al. Ultrathin $\mathrm{CeO}_{2}$ coating for improved cycling and rate performance of Ni-rich layered $\mathrm{LiNi}_{0.7} \mathrm{Co}_{0.2} \mathrm{Mn}_{0.1} \mathrm{O}_{2}$ cathode materials. Ceram. Int., 2019, 45(1): 144-152.

[106] CHEN Y X, LI Y J, LI W, et al. High-voltage electrochemical performance of $\mathrm{LiNi}_{0.5} \mathrm{Co}_{0.2} \mathrm{Mn}_{0.3} \mathrm{O}_{2}$ cathode material via the synergetic modification of the $\mathrm{Zr} / \mathrm{Ti}$ elements. Electrochim. Acta, 2018, 281: 48-59.

[107] DU R, BI Y, YANG W, et al. Improved cyclic stability of $\mathrm{LiNi}_{0.8} \mathrm{Co}_{0.1} \mathrm{Mn}_{0.1} \mathrm{O}_{2}$ via Ti substitution with a cut-off potential of 4.5 V. Ceram. Int., 2015, 41(5): 7133-7139.

[108] PARK S H, OH S W, SUN, Y K. Synthesis and structural characterization of layered $\mathrm{Li}\left[\mathrm{Ni}_{1 / 3+x} \mathrm{Co}_{1 / 3} \mathrm{Mn}_{1 / 3-2 x} \mathrm{Mo}_{x}\right] \mathrm{O}_{2}$ cathode materials by ultrasonic spray pyrolysis. J. Power Sources, 2005, 146(1/2): 622-625.

[109] YANG J, XIA Y Y. Suppressing the phase transition of the layered Ni-rich oxide cathode during high-voltage cycling by introducing low-content $\mathrm{Li}_{2} \mathrm{MnO}_{3}$. ACS Appl. Mater. Interfaces, 2016, 8(2): 1297-1308.

[110] BAK S M, NAM K W, CHANG W, et al. Correlating structural changes and gas evolution during the thermal decomposition of charged $\mathrm{Li}_{x} \mathrm{Ni}_{0.8} \mathrm{Co}_{0.15} \mathrm{Al}_{0.05} \mathrm{O}_{2}$ cathode materials. Chem. Mater., 2013, 25(3): 337-351.

[111] MIN K, SEO S W, SONG Y Y, et al. A first-principles study of the preventive effects of $\mathrm{Al}$ and $\mathrm{Mg}$ doping on the degradation in $\mathrm{LiNi}_{0.8} \mathrm{Co}_{0.1} \mathrm{Mn}_{0.1} \mathrm{O}_{2}$ cathode materials. Phys. Chem. Chem. Phys., 2017, 19: 1762-1769.

[112] DIXIT M, MARKOVSKY B, AURBACH D, et al. Unraveling the effects of $\mathrm{Al}$ doping on the electrochemical properties of $\mathrm{LiNi}_{0.5} \mathrm{Co}_{0.2} \mathrm{Mn}_{0.3} \mathrm{O}_{2}$ using first principles. J. Electrochem. Soc., 2017, 164(1): A6359-A6365.

[113] XIAO P H, DENG Z Q, MANTHIRAM A, et al. Calculations of oxygen stability in lithium-rich layered cathodes. J. Phys. Chem. C, 2012, 116(44): 23201-23204.

[114] AURBACH D, SRUR-LAVI O, GHANTY C, et al. Studies of aluminum-doped $\mathrm{LiNi}_{0.5} \mathrm{Co}_{0.2} \mathrm{Mn}_{0.3} \mathrm{O}_{2}$ : electrochemical behavior, aging, structural transformations, and thermal characteristics. $J$. Electrochem. Soc., 2015, 162(6): A1014-A1027.

[115] KIM U H, JUN D W, PARK K J, et al. Pushing the limit of layered transition metal oxide cathodes for high-energy density rechargeable Li ion batteries. Energy Environ. Sci., 2018, 11: 1271-1279.

[116] KONISHI H, YOSHIKAWA M, HIRANO T. The effect of thermal stability for high-Ni-content layer-structured cathode materials, $\mathrm{LiNi}_{0.8} \mathrm{Mn}_{0.1-x} \mathrm{Co}_{0.1} \mathrm{Mo}_{x} \mathrm{O}_{2}(x=0,0.02,0.04)$. J. Power Sources, 2013, 244: 23-28.

[117] LIU Q, ZHAO Z K, WU F, et al. The effects of molybdenum doping on $\mathrm{LiNi}_{0.6} \mathrm{Co}_{0.2} \mathrm{Mn}_{0.2} \mathrm{O}_{2}$ cathode material. Solid State Ionics, 2019, 337: 107-114.

[118] WEIGEL T, SCHIPPER F, ERICKSON E M, et al. Structural and electrochemical aspects of $\mathrm{LiNi}_{0.8} \mathrm{Co}_{0.1} \mathrm{Mn}_{0.1} \mathrm{O}_{2}$ cathode materials doped by various cations. ACS Energy Lett., 2019, 4(2): 508-516.

[119] XIANG W, ZHU C Q, ZHANG J, et al. Synergistic coupling effect of sodium and fluorine co-substitution on enhancing rate capability and cycling performance of Ni-rich cathode for lithium ion battery. J. Alloys Compd., 2019, 786: 56-64.

[120] HU G, ZHANG M, LIANG L, et al. Mg-Al-B co-substitution $\mathrm{LiNi}_{0.5} \mathrm{Co}_{0.2} \mathrm{Mn}_{0.3} \mathrm{O}_{2}$ cathode materials with improved cycling performance for lithium-ion battery under high cutoff voltage. Electrochim. Acta, 2016, 190: 264-275.

[121] CHANG S H, CHEN Y X, LI Y J, et al. Improvement of the 
high-voltage electrochemical properties of $\mathrm{Li}\left[\mathrm{Ni}_{0.5} \mathrm{Co}_{0.2} \mathrm{Mn}_{0.3}\right] \mathrm{O}_{2} @$ $\mathrm{ZrO}_{2}$ cathode materials with liquid phase modification. J. Alloys Compd., 2019, 781: 496-503.

[122] LI X, ZHANG K J, WANG M S, et al. Dual functions of zirconium modification on improving the electrochemical performance of Ni-rich $\mathrm{LiNi}_{0.8} \mathrm{Co}_{0.1} \mathrm{Mn}_{0.1} \mathrm{O}_{2}$. Sustain. Energ. Fuels, 2018, 2: 413-421.

[123] HE T, LU Y, SU Y F, et al. Sufficient utilization of zirconium ions to improve the structure and surface properties of nickel-rich cathode materials for lithium-ion batteries. ChemSusChem, 2018, 11(10): $1639-1648$.

[124] SUN Y K, KIM D H, YOON C S, et al. A novel cathode material with a concentration-gradient for high-energy and safe lithiumion batteries. Adv. Funct. Mater., 2010, 20(3): 485-491.

[125] XU X, JIAN J Y, XIANG L Z, et al. Enhancing high-voltage performances of nickel-based cathode material via aluminum and progressive concentration gradient modification. Electrochim. Acta, 2019, 317: 459-467.

[126] SHI J L, QI R, ZHANG X D, et al. High-thermal- and air-stability cathode material with concentration-gradient buffer for Li-ion batteries. ACS Appl. Mater. Interfaces, 2017, 9(49): 42829-42835.

[127] TANG M J, YANG J, CHEN N T, et al. Overall structural modification of a layered $\mathrm{Ni}$-rich cathode for enhanced cycling stability and rate capability at high voltage. J. Mater. Chem. A, 2019, 7: 6080-6089.

[128] RAN Q W, ZHAO H Y, WANG Q, et al. Dual functions of gradient phosphate polyanion doping on improving the electrochemical performance of Ni-rich $\mathrm{LiNi}_{0.6} \mathrm{Co}_{0.2} \mathrm{Mn}_{0.2} \mathrm{O}_{2}$ cathode at high cut-off voltage and high temperature. Electrochim. Acta, 2019, 299: 971-978.

[129] ZHANG M L, ZHAO H Y, TAN M, et al. Yttrium modified Ni-rich $\mathrm{LiNi}_{0.8} \mathrm{Co}_{0.1} \mathrm{Mn}_{0.1} \mathrm{O}_{2}$ with enhanced electrochemical performance as high energy density cathode material at $4.5 \mathrm{~V}$ high voltage. J. Alloys Compd., 2019, 774: 82-92.

[130] TANG W J, CHEN Z X, XIONG F, et al. An effective etchinginduced coating strategy to shield $\mathrm{LiNi}_{0.8} \mathrm{Co}_{0.1} \mathrm{Mn}_{0.1} \mathrm{O}_{2}$ electrode materials by $\mathrm{LiAlO}_{2}$. J. Power Sources, 2019, 412: 246-254.

[131] YU H F, LI Y G, HU Y J, et al. 110th anniversary: concurrently coating and doping high-valence vanadium in nickel-rich lithiated oxides for high-rate and stable lithium-ion batteries. Ind. Eng. Chem. Res., 2019, 58(10): 4108-4115.

[132] CHEN Y X, LI Y J, TANG S Y, et al. Enhanced electrochemical properties of the Cd-modified $\mathrm{LiNi}_{0.6} \mathrm{Co}_{0.2} \mathrm{Mn}_{0.2} \mathrm{O}_{2}$ cathode materials at high cut-off voltage. J. Power Sources, 2018, 395: 403-413.

[133] KONG D F, HU J T, CHEN Z F, et al. Ti-gradient doping to stabi- lize layered surface structure for high performance high-Ni oxide cathode of Li-ion battery. Adv. Energy Mater., 2019, 9: 1901756.

[134] HAN B, XU S, ZHAO S, et al. Enhancing the structural stability of Ni-rich layered oxide cathodes with a preformed $\mathrm{Zr}$-concentrated defective nanolayer. ACS Appl. Mater. Interfaces, 2018, 10(46): 39599-39607.

[135] SCHIPPER F, BOUZAGLO H, DIXIT M, et al. From surface $\mathrm{ZrO}_{2}$ coating to bulk $\mathrm{Zr}$ doping by high temperature annealing of nickel-rich lithiated oxides and their enhanced electrochemical performance in lithium ion batteries. Adv. Energy Mater., 2018, 8(4): 1701682.

[136] MENG K, WANG Z X, GUO H J, et al. Improving the cycling performance of $\mathrm{LiNi}_{0.8} \mathrm{Co}_{0.1} \mathrm{Mn}_{0.1} \mathrm{O}_{2}$ by surface coating with $\mathrm{Li}_{2} \mathrm{TiO}_{3}$. Electrochim. Acta, 2016, 211: 822-831.

[137] WU F, LI Q, CHEN L, et al. Use of Ce to reinforce the interface of $\mathrm{Ni}$-rich $\mathrm{LiNi}_{0.8} \mathrm{Co}_{0.1} \mathrm{Mn}_{0.1} \mathrm{O}_{2}$ cathode materials for lithium-ion batteries under high operating voltage. ChemSusChem, 2019, 12(4): 935-943.

[138] ZHAN X W, GAO S, CHENG Y T. Influence of annealing atmosphere on $\mathrm{Li}_{2} \mathrm{ZrO}_{3}$-coated $\mathrm{LiNi}_{0.6} \mathrm{Co}_{0.2} \mathrm{Mn}_{0.2} \mathrm{O}_{2}$ and its high-voltage cycling performance. Electrochim. Acta, 2019, 300: 36-44.

[139] 李宁, 李文进, 张宇宙, 等. 一种梯度高镍正极材料及其制备 方法和锂离子电池. 中国, CN107528060A. 2017.12.29.

[140] WANG D, LI X H, WANG Z X, et al. Co-modification of $\mathrm{LiNi}_{0.5} \mathrm{Co}_{0.2} \mathrm{Mn}_{0.3} \mathrm{O}_{2}$ cathode materials with zirconium substitution and surface polypyrrole coating: towards superior high voltage electrochemical performances for lithium ion batteries. Electrochim. Acta, 2016, 196: 101-109.

[141] YANG H P, WU H H, GE M Y, et al. Simultaneously dual modification of Ni-rich layered oxide cathode for high-energy lithiumion batteries. Adv. Funct. Mater., 2019, 29(13): 1808825.

[142] RAN Q W, ZHAO H Y, SHU X H, et al. Enhancing the electrochemical performance of Ni-rich layered oxide cathodes by combination of the gradient doping and dual-conductive layers coating. ACS Appl. Energy Mater., 2019, 2(5): 3120-3130.

[143] SUN S M, LIU T, NIU Q H, et al. Improvement of superior cycle performance of $\mathrm{LiNi}_{0.8} \mathrm{Co}_{0.15} \mathrm{Al}_{0.05} \mathrm{O}_{2}$ cathode for lithium-ion batteries by multiple compound modifications. J. Electroanal. Chem., 2019, 838: 178-185.

[144] 任志敏, 王振尧, 高敏, 等. 一种磷镁协同掺杂改性的富锂锰 基正极材料及其制备方法和锂离子电池.中国, CN107591534A. 2018.01.16.

[145] XU G L, LIU Q, LAU K K S, et al. Building ultraconformal protective layers on both secondary and primary particles of layered lithium transition metal oxide cathodes. Nat. Energy, 2019, 4: 484-494. 